\title{
Nanoscale
}

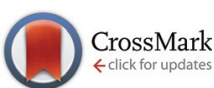

Cite this: Nanoscale, 2016, 8, 4414

Received 8th September 2015, Accepted 24th November 2015

DOI: $10.1039 /$ c5nr06175j

www.rsc.org/nanoscale

\section{Carbon-atom wires: 1-D systems with tunable properties}

\author{
C. S. Casari, ${ }^{\star a}$ M. Tommasini, ${ }^{b}$ R. R. Tykwinski ${ }^{c}$ and A. Milani ${ }^{b}$
}

This review provides a discussion of the current state of research on linear carbon structures and related materials based on sp-hybridization of carbon atoms (polyynes and cumulenes). We show that such systems have widely tunable properties and thus represent an intriguing and mostly unexplored field for both fundamental and applied sciences. We discuss the rich interplay between the structural, vibrational, and electronic properties focusing on recent advances and the future perspectives of carbon-atom wires and novel hybrid $\mathrm{sp}-\mathrm{sp}^{2}$-carbon architectures.

\section{Introduction}

Carbon is a chemically versatile element that can adopt different hybridization states to produce a wide variety of allotropic forms, molecules, and nanostructures. sp-, $\mathrm{sp}^{2}-$, and $\mathrm{sp}^{3}$-hybridized atomic orbitals give rise to linear, planar and three-dimensional organization in carbon-based molecules, (e.g., alkynes, alkenes, and alkanes respectively) as well as in

\footnotetext{
${ }^{a}$ Department of Energy, Politecnico di Milano, via Ponzio 34/3, 20133 Milano, Italy. E-mail: carlo.casari@polimi.it; Fax: (+39) 0223996309; Tel: (+39) 0223996331

${ }^{b}$ Department of Chemistry, Materials and Chemical Engineering 'G. Natta', Politecnico di Milano, Piazza Leonardo da Vinci 32, 20133 Milano, Italy ${ }^{c}$ Department of Chemistry and Pharmacy \& Interdisciplinary Center for Molecular Materials (ICMM), Friedrich-Alexander-Universität Erlangen-Nürnberg (FAU), Henkestrasse 42, 91054 Erlangen, Germany
}

condensed matter (isotropic and anisotropic structures of diamond and graphite, respectively). A crystalline solid made of sp-carbon is absent as a third allotropic form of carbon, whose existence is questionable or even considered impossible for stability reasons. Low-dimensional carbon structures comprise quasi-zero- and quasi-one-dimensional (quasi-0-D and quasi-1-D) systems such as fullerenes, carbon clusters, nanodiamonds, and nanotubes, as well as graphene as a twodimensional (2-D) system. Over the last 30 years carbon nanostructures have indeed played a leading role in nanoscience and nanotechnology. ${ }^{1,2}$ Fullerenes, nanotubes, and graphene demonstrate that carbon can form a wide variety of structures whose properties are strongly related to structural issues such as dimensionality, hybridization, chirality and topology. Such structures are based on $\mathrm{sp}^{3}-\mathrm{sp}^{2}$-, or mixed $\mathrm{sp}^{2}-\mathrm{sp}^{3}$-carbon hybridization as shown in the ternary diagram of Fig. 1.

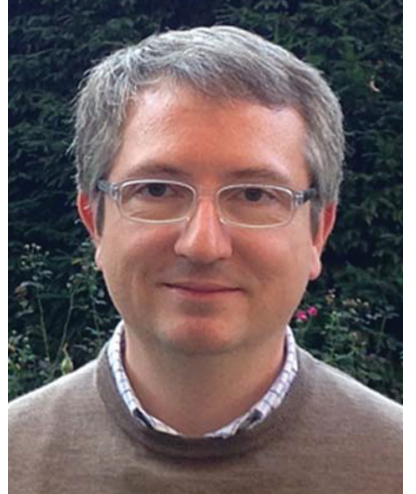

M. Tommasini
Matteo Tommasini was born in 1972 in Desenzano del Garda (Brescia, Italy). At Politecnico di Milano (Italy) he graduated in Nuclear Engineering in 1998 and he obtained his PhD in Materials Engineering in 2002 under the guidance of Prof. G. Zerbi and Prof. C. Castiglioni. He is associate professor in Materials Science and Technology at Politecnico di Milano. His research interest focuses on the applications of Vibrational Spectroscopy and Quantum Chemistry to Materials Science and Biotechnology. 


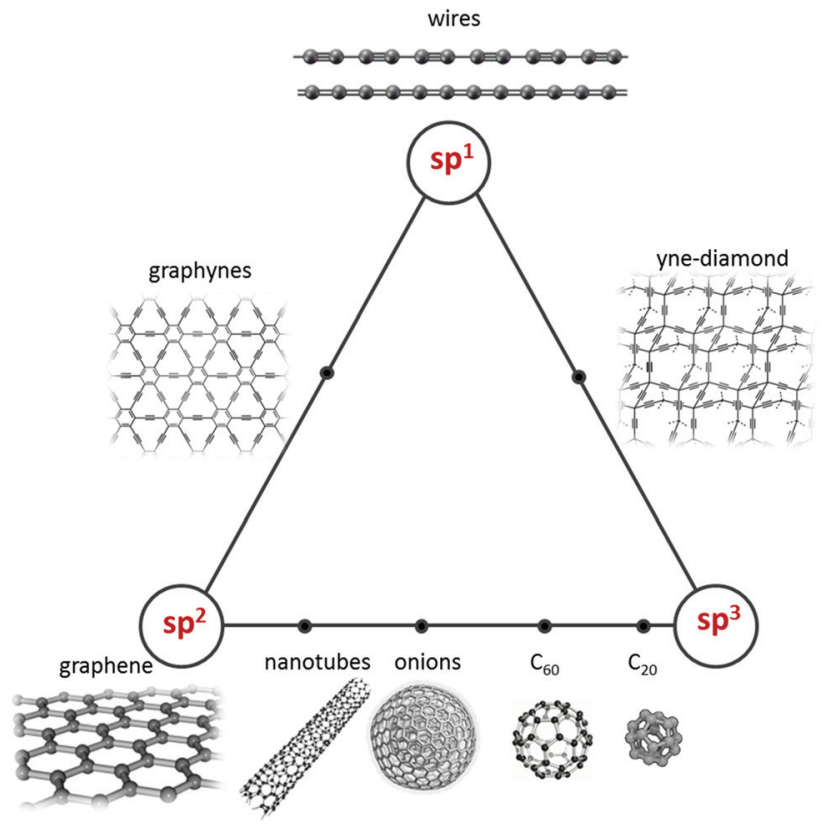

Fig. 1 Ternary diagram of carbon nanostructures according to their hybridization state. Adapted with permission from C. S. Casari Istituto Lombardo (Rend. Scienze), 2012, 146, 17-35 [eISSN 2384-986X].

Following this classification, the sp-vertex is occupied by linear nanostructures and molecules in the form of carbon-atom wires (CAWs). Other possible novel structures that have been predicted can be placed along the lines connecting sp- with $\mathrm{sp}^{2}$ - and $\mathrm{sp}^{3}$-carbon, namely hybrid systems of $\mathrm{sp}-\mathrm{sp}^{2}$-carbon (e.g., graphyne and graphdiyne) and $\mathrm{sp}^{-\mathrm{sp}^{3}}$ carbon (forms of 'superdiamond' such as yne-diamond). ${ }^{1}$

Importantly, sp- and $\mathrm{sp}^{2}$-hybridized carbon systems can sustain $\pi$-conjugation, which is the basis of the metallic pro- perties of graphene and carbon nanotubes. Graphene is a zero-gap material with outstanding electronic and optical properties for advanced technological applications (e.g., extremely high electron mobility, ambipolar conductivity related to Dirac's cones, and optical transparency). ${ }^{3-5}$

Opening a band gap in graphene requires additional strategies such as the exploitation of confinement effects, as it happens in graphene nanoribbons. In carbon nanotubes, the electronic properties, including the semiconducting behaviour, are dominated by topological issues defined by the choice of the chiral vector and can thus be tuned to some extent. ${ }^{6}$ However the control of the chirality is still an open issue, which makes it difficult to tune the electronic properties of these systems.

As graphene is today considered the ultimate 2-dimensional (2-D) system (1-atom-thick) showing peculiar electronic properties, CAWs represent a true 1-D system (1-atomdiameter) that might display either semiconducting or metallic properties due to $\pi$-conjugation effects. ${ }^{7,8}$ In fact, in an extended linear sp-carbon system forming a CAW, the semiconductive/metallic behaviour results from the trade-off between Peierls distortion (which favours semiconductivity) and endgroup effects, which can stabilize metallic structures. Hence, sp-hybridized CAWs are intriguing systems with structure-, length-, and endgroup-dependent properties. A model CAW can show two distinct structures: $=\mathrm{C}=\mathrm{C}=\mathrm{C}=\mathrm{C}=$ and $-\mathrm{C} \equiv \mathrm{C}-\mathrm{C} \equiv \mathrm{C}-\mathrm{C} \equiv$ (see Fig. 2). In real systems, the finite

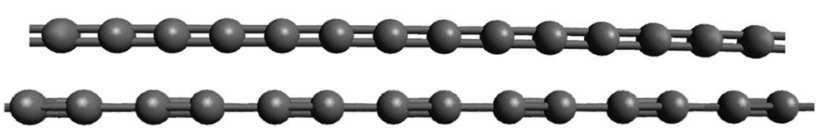

Fig. 2 The two possible structures of the ideal infinite carbon-atom wire (carbyne): an equalized structure with double bonds (cumulene, top) and alternate single-triple bonds (polyyne, bottom).

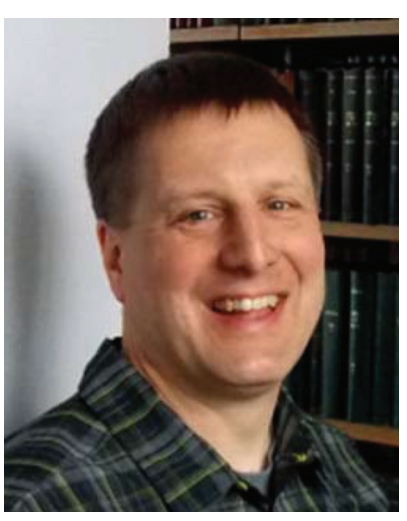

R. R. Tykwinski
Rik R. Tykwinski was born in 1965 in Marshall, MN. He obtained his BS degree (1987) from the University of Minnesota - Duluth and his PhD (1994) from the University of Utah. He moved to ETH-Zürich for his postdoctoral research (1994-1997), and in 1997 he joined the faculty at the University of Alberta. In 2009, he moved to Germany and began as Chair of Organic Chemistry at the Friedrich-AlexanderUniversität Erlangen-Nürnberg (FAU). His interests focus on the development of synthetic methods for carbon-rich molecules and allotropes, the electronic properties of carbon-rich molecules, and applications of conjugated systems to molecular electronics, as well as mountain biking and entertaining his two sons.

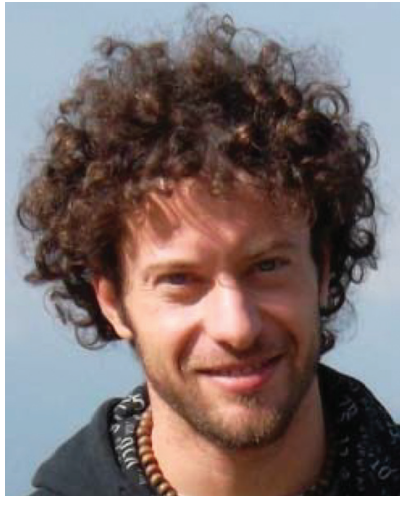

Alberto Milani was born in 1980 in Erba - Italy. In 2005, he graduated in Materials Engineering at Politecnico di Milano where he also obtained a PhD in Materials Engineering in April 2009. Since 2005, he joined the research group led by Prof. Zerbi and Prof. Castiglioni as assistant professor and researcher. His scientific interest focuses on computational materials science and vibrational spectroscopy, in particular on the application of state-of-the-art quantum chemical methods for the investigation of the spectroscopic response of polyconjugated molecules and polymers. 
length effects and endgroups play a fundamental role and drive the system to a specific degree of bond length alternation: the terminal groups affect the overall wire configuration, with an increasingly larger effect in shorter wires. ${ }^{8}$ Control of the electronic properties (band gap and conducting character) by tuning of the wire structure may thus open new perspectives for the realization of nanoscale cables and devices, as shown by theoretical predictions but not yet fully assessed by experiments. $^{9-11}$

Since the first understanding that diamond and graphite were made of the same "substance" (experimentally demonstrated by S. Tennant in 1796), ${ }^{12}$ scientists have been looking for novel carbon allotropes, including the linear forms of sphybridized carbon. The claims of the discovery of a new allotrope based on sp-hybridized carbon, namely carbyne, (the mineral form found in a meteor crater in Germany was called Chaoite) date back to the $1960 \mathrm{~s},{ }^{13-16}$ but were never confirmed even after intense debates following some criticisms raised in the 1980s. ${ }^{17-19}$ In the same years, astrophysics studies focused on the possible presence of linear carbon in the interstellar medium, and such studies led to the discovery of fullerenes by Kroto, Smalley and Curl. ${ }^{20}$ Recently, new achievements and the ground-breaking results on graphene have again driven the attention to CAWs and sp-sp ${ }^{2}$-hybrids (e.g., graphyne) as novel 1-D and 2-D systems. Hence, the general skepticism about the existence of a third solid allotrope of carbon has been replaced by the interest in 1-D and 2-D nanoscale sp-carbon structures. ${ }^{1,21}$ Nowadays the occurrence of sp-hybridized carbon has been suggested in many carbon-based materials and structures, in carbon-metal moieties, embedded in matrices, after laser irradiation of polymers, in free carbon clusters, in pure $\mathrm{sp}^{-\mathrm{sp}^{2}}$ systems, in liquids, inside carbon nanotubes (CNTs) and connecting graphene sheets. $^{22-35}$ In addition, synthetic strategies allow us to produce long and stable sp-carbon wires with controlled length and termination. ${ }^{36}$ Theoretical investigations have predicted a number of interesting vibrational, electronic, and transport properties that can be tuned by controlling the wire length and endgroups. This includes carbon wires connecting metal leads, graphene, and nanotubes as device prototypes. $^{37-47}$

Here we review the present state of research on carbonatom wires based on both experimental and theoretical results, with particular focus on the structure-dependent properties. The review is organized as follows. We start by discussing the structure of the ideal model system (i.e., an infinite wire as a 1-D crystal), the related $\pi$-conjugation effects and the change in the electronic properties as a result of the wire length and termination. Then we will review synthesis techniques toward CAWs and strategies to improve stability. We will discuss the present understanding of the structure of CAWs that mainly results from Raman and surface enhanced Raman scattering (SERS) characterization of selected CAW systems. The electronic and transport properties are then discussed within the perspectives of the use of CAWs in advanced nanotechnology applications.

\section{Structural, vibrational, and electronic properties of carbon-atom wires}

To discuss the structure of CAWs, it is better first to clarify the existing nomenclature of sp-carbon since it is rather diversified and not fully settled. In the past, the name carbyne was used to indicate the solid form of sp-carbon while Chaoite or white carbon has been used for minerals or solid samples found or synthesized in laboratory. ${ }^{13}$ More recently, the name carbyne has been adopted to indicate an infinite sp-carbon wire as a model system. Similarly, carbynoid structures have been used to indicate finite systems or moieties comprising sp-carbon in amorphous systems. Different names have been adopted to name the isolated systems, e.g., linear carbon chains, carbon monatomic chains or carbon atomic chains. Here, we will refer to all these systems as carbon-atom wires - CAWs. When needed, the distinction between ideal (infinite) and real (finite) CAWs will be stated.

\subsection{The ideal infinite wire: interplay between electronic and vibrational properties}

Despite the apparent simplicity of a system composed by sphybridized carbon atoms arranged in a linear (or almost linear) geometry, the physico-chemical properties of CAWs are far from trivial, as discussed in the following section. As a first approximation, the physical properties of CAWs can be interpreted on the basis of the model of an infinite chain of carbon atoms (carbyne). This ideal model allows investigation of CAWs following a convenient solid-state physics approach, similarly to other polymeric molecular materials. ${ }^{48-53}$ On these grounds, even if some endgroup effects necessarily go beyond the infinite chain model, we will show that the properties of finite length sp-carbon wires can be still discussed with reference to this simple model. ${ }^{5,55}$ When needed this somehow restrictive approximation can be relaxed.

In contrast to "classical" $\sigma$-bonded polymers (e.g., polyethylene), due to $\pi$-conjugation and electron-phonon coupling, the connection between finite and infinite wires is not straightforward in CAWs. In fact, starting with the infinite structure, two arrangements of the $\mathrm{C}$ atoms within the unit cell are possible (Fig. 2).

One structure consists of equivalent (equalized) double bonds. This is referred to as polycumulene (or simply cumulene) and it can be described as a 1-D crystal with a monoatomic unit cell. The other arrangement is characterized by a sequence of alternating triple and single bonds (polyyne). The polyyne form corresponds to a 1-D crystal with a unit cell containing two carbon atoms. Cumulene is a metal since the conduction band is half filled (one carbon atom contributes to one electron for each of the two $2 p$ orbitals). In polyynes, however, the double unit cell provides two electrons in each orbital and results in a half Brillouin zone. Hence, the valence band is completely filled by electrons while the conduction band, separated by the band gap opened at the edge of the Brillouin zone, is empty thus leading to a semiconductor 
(Fig. 3). Due to $\pi$-electron delocalization, the CC bond lengths of the infinite polyyne are not exactly those of an ordinary triple and a single CC bond (e.g., that of acetylene or ethane). Actually the triple bonds are slightly longer than that in acetylene ( $c a .1 .2 \AA)$ and the single bonds are slightly shorter than that in ethane ( $c a .1 .5 \AA)$. A useful parameter to define the wire structure is bond length alternation (BLA), which is the difference in bond lengths for adjacent bonds (typically measured at the centre of the molecule). BLA is non-zero in polyyne, ${ }^{56}$ and it is ideally zero for cumulene. As in the case of a linear metal and other polyconjugated materials (e.g., polyacetylene), CAWs are affected by Peierls distortion, ${ }^{57,58}$ which states that cumulene is unstable upon a change in BLA and undergoes a transition to the alternated, polyyne-like configuration that corresponds to minimum energy. This is illustrated in Fig. 4
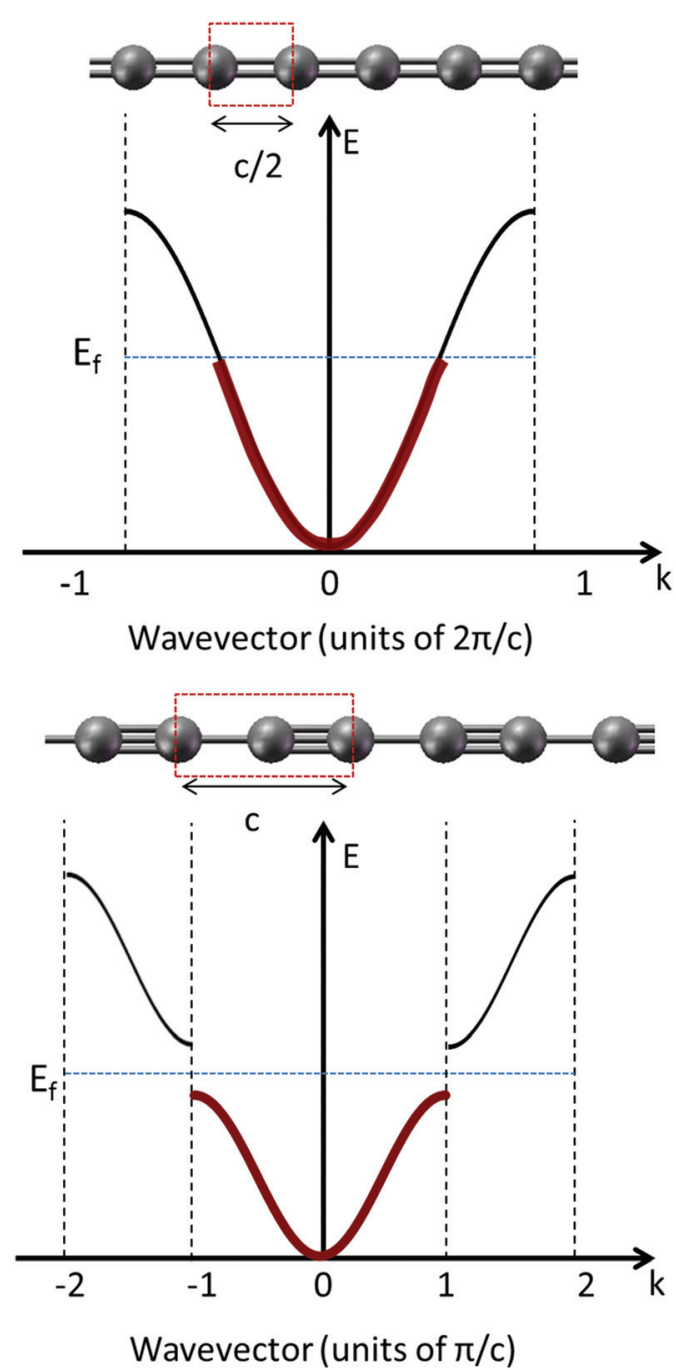

Fig. 3 Sketch of the two possible geometric arrangements of the infinite CAW (i.e., carbyne) with the corresponding band structure according to a solid-state physics approach: an equalized double-bond structure (polycumulene, top), and alternate quasi simple-quasi triple bonds (polyyne, bottom). The band filling reveals the metal and semiconducting character of cumulene and polyyne, respectively.

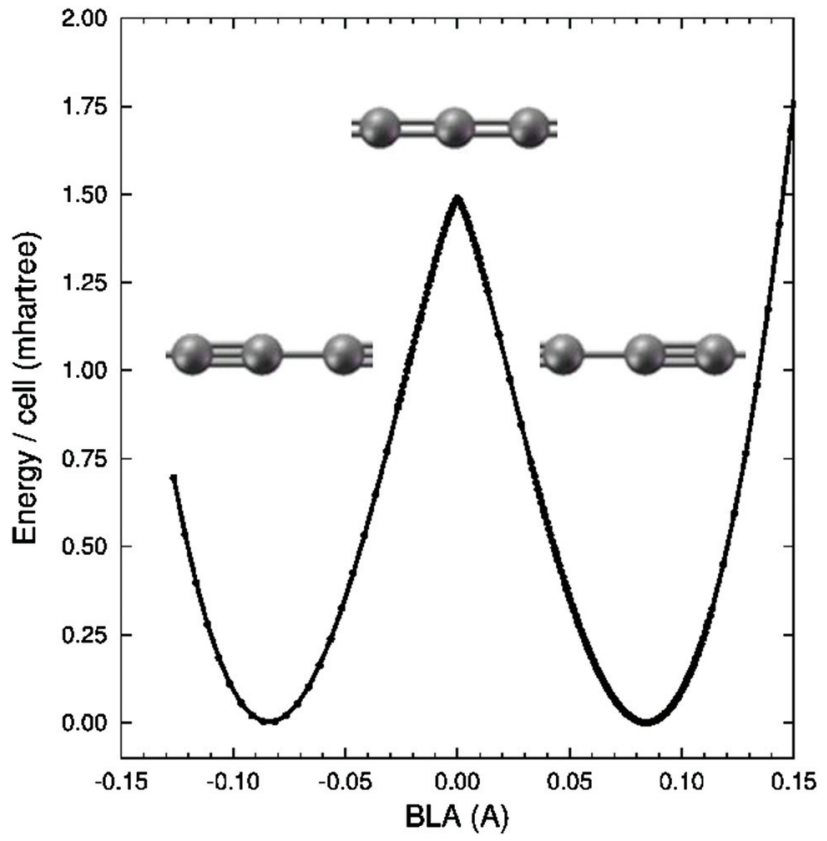

Fig. 4 Potential energy surface of an isolated infinite linear carbon chain as a function of BLA, as obtained by periodic boundary condition calculations, ${ }^{59}$ showing the occurrence of Peierls distortion (instability of the equalized, cumulene-like geometry) and the stabilization of the two possible (and equivalent) bond alternated (polyyne-like) structures.

by the potential energy curve plotted as a function of BLA, as obtained by quantum chemical calculations. ${ }^{59}$

BLA significantly affects the electronic structure of the spcarbon chain, driving the transition from a metallic to a semiconducting system, which influences both the optical and vibrational properties. This transition is illustrated by the electronic band structure of ideal CAWs as a function of BLA (Fig. 5) resulting from DFT pseudopotential calculations. ${ }^{54}$ As
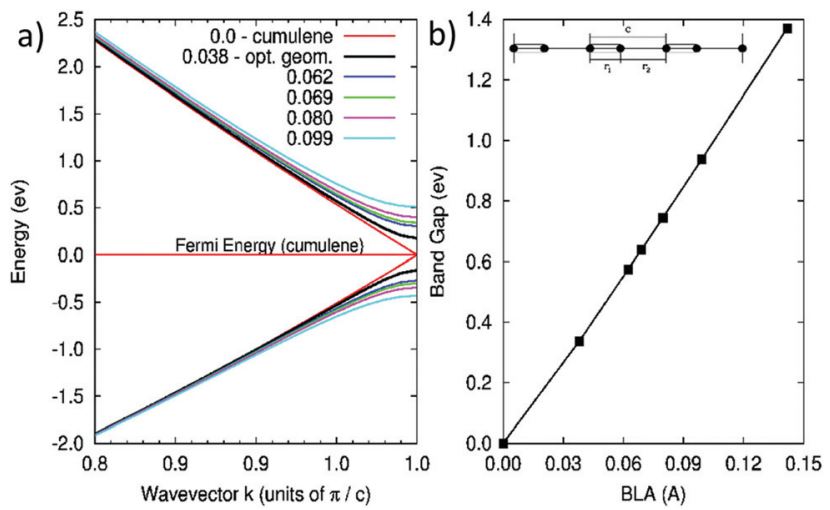

Fig. 5 (a) Band structure of a carbon atomic wire for cumulene (BLA = 0 ) and for different values of BLA in Å (ideal polyyne with an optimized geometry shows a BLA $=0.038 \AA$ ); adapted with permission from ref. 54 , copyright 2006 by the American Physical Society. (b) Band gap as a function of BLA. The wire structure and definition of BLA as $r_{2}-r_{1}$ is also shown. 
required by Peierls distortion, the equilibrium geometry has BLA $\neq 0$, corresponding to a finite band gap. The band gap widens for increasing BLA, while it decreases for smaller BLA. In the limit of small BLA, $\pi$-electron conjugation is enhanced, i.e., the electrons are more effectively delocalized along the chain, until the metallic character is actually reached in cumulene $(\mathrm{BLA}=0)$. As a consequence of Peierls distortion, only polyyne chain represents the minimum energy configuration leading to a well-defined BLA value (i.e., corresponding to an optimized geometry). Other cases obtained by imposing a different geometry may represent convenient models to investigate the effects of modulating the BLA. In fact, when relaxing the infinite chain approximation, we can see that the endgroup effects, charge transfer, photoexcitation processes, etc. are all effective ways of modulating the BLA. Hence, real systems (finite length CAWs) can be correlated with the appropriate model of an infinite chain possessing the same BLA. The possibility of inducing, by means of chemical or physical processes, an effective modulation of the molecular structure that can control the electronic properties is very appealing from the point of view of the possible applications of CAWs in technology.

Due to the well-known electron-phonon coupling that exists in all $\pi$-conjugated systems, it is reasonable to expect that changes in BLA might also significantly influence the vibrational properties of CAWs. This motivates the use of vibrational spectroscopy (IR and Raman) as a sensitive method for characterization of sp-carbon wires (see section 2.3).

In order to discuss the vibrational structure of CAWs, it is useful to refer to the limiting case of the infinite linear carbon chain. Considering cumulene first, the description of its phonon dispersion is elementary and constitutes a classical textbook example for introducing the concept of phonons in a monoatomic infinite chain as a 1-D crystal. ${ }^{60}$ Cumulene displays only acoustic phonon branches (one longitudinal acoustical, 1LA and 2TA, respectively), and the system would not present any optical activity. ${ }^{57,60}$ The polyyne case corresponds to a homo-atomic chain with different bond strengths so that the unit cell is composed by two atoms. As a result, there are three acoustic and three optical phonon branches (Fig. 6). In other words, upon moving from cumulene to polyyne the major effect is the appearance of optical phonon branches. This matter has been considered in the literature to exclude the possibility that cumulene-like sp-carbon wires might not be observed by Raman or IR spectroscopy. While this is certainly true for infinite cumulene, for finite length CAWs this selection rule must be relaxed, since endgroups play a role, i.e., BLA is affected by the terminal endcapping groups, and the spectroscopic behaviour of finite-length cumulenes will deviate from the infinite chain model. ${ }^{61}$

The phonon dispersion of the longitudinal optical (LO) branch has been computed for polyynes with varying BLA by means of a tight-binding model that couples the vibrational force field (i.e., the dynamical matrix) to the expected changes of the electronic structure. ${ }^{54}$ This approach corresponds to solving the vibrational dynamics of the CAW subjected to a
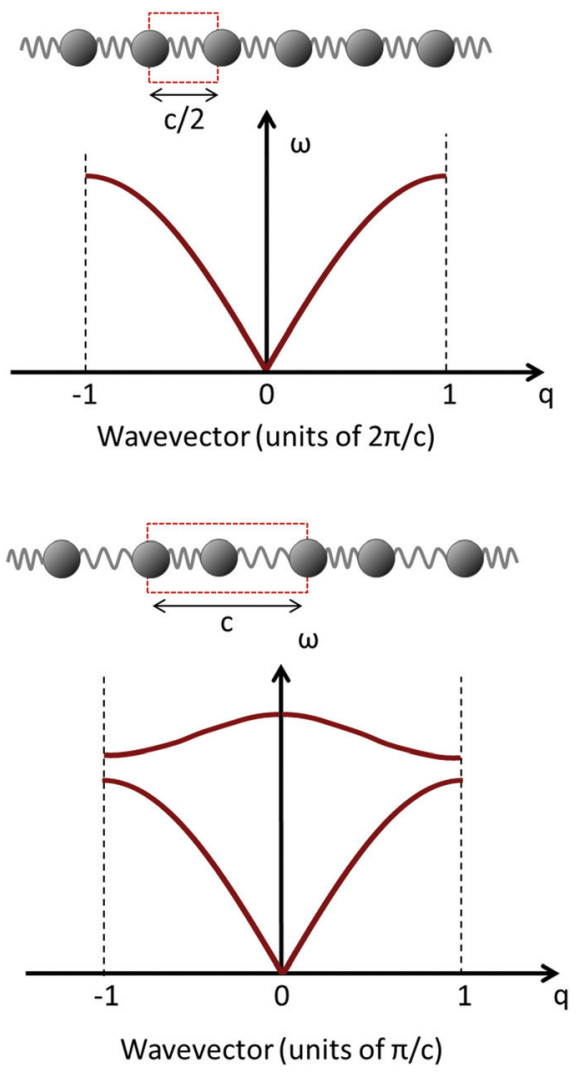

Fig. 6 Sketch of the phonon dispersion branches of a bond equalized (cumulene, top) and alternate (polyyne, bottom) infinite wire (i.e., carbyne as a 1-D crystal).

constant external force that sets the BLA as desired. As shown in Fig. 7, the LO branch depends dramatically on BLA: the phonon wavenumber at $q=0$ ranges from 1200 to more than $2000 \mathrm{~cm}^{-1}$ for increasing BLA in the range 0.038-0.142 $\AA^{54}$
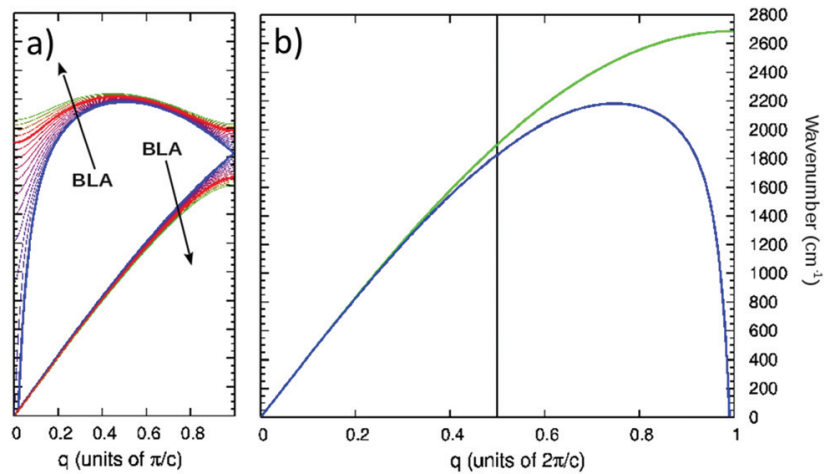

Fig. 7 Phonon dispersion relationship for wires as a function of BLA. (a) Longitudinal optical (LO) and longitudinal acoustic (LA) phonon dispersion branches of an infinite polyyne (adapted with permission from ref. 55, copyright 2008 AIP Publishing LLC). (b) Comparison between phonon dispersion of a carbon-atom wire with (green line) and without (blue line) the nearest neighbour approximation. 
The over bending of the phonon dispersion and the softening of the phonon in the vicinity of $q=0$ are due to long-range vibrational interactions along the chain ${ }^{55,62}$ (i.e., the inclusion in the harmonic potential of interactions beyond the nearest neighbour approximation). ${ }^{59}$ The strength of long-range interactions increases as the system approaches the structure of cumulene, and the electronic structure evolves from semiconducting to metallic. In fact, similarly to graphite ${ }^{63}$ and carbon nanotubes, ${ }^{64,65}$ cumulene is affected by a Kohn anomaly ${ }^{54}$ that makes the phonon dispersion divergent at the $\Gamma$ point (in the reciprocal space of polyyne).

As shown in Fig. 7b, which depicts the acoustic phonon branch of cumulene, if one considers only the nearest neighbour interactions in the harmonic potential, one can fit only the frequencies close to the $\Gamma$ point, where the Kohn anomaly effects are negligible. This approximation breaks down at the first Brillouin zone boundary, which shows that the Kohn anomaly is due to the effects beyond the nearest neighbour approximation and may also require inclusion of anharmonicity. It is important to note that by taking the description of cumulene with a translational unit comprised of two carbon atoms (Fig. 7a), the Kohn anomaly is found at $q=0$, otherwise it is found at the Brillouin zone boundary (Fig. 7b).

To gain better insight into the vibrational properties of CAWs, it is worth introducing some additional theoretical details, which are based upon theories introduced to deal with the vibrational spectroscopy of $\pi$-conjugated polymers. ${ }^{6-70}$ In this field, Raman spectroscopy certainly plays a major role (see for instance ref. 71-75 and references therein). The Raman spectra of $\pi$-conjugated polymers are usually dominated by intense Raman lines, which are related to BLA oscillation. These lines show a sizeable frequency and intensity dispersion with the chain length. For increasing chain length the Raman signal associated with BLA oscillation shifts to lower wavenumbers and becomes more intense. This behaviour has been rationalized by means of two theories, the "amplitude mode theory" $(\mathrm{AMT})^{70}$ and the "effective conjugation coordinate" (ECC) model. ${ }^{71-74}$ These two approaches are rigorously related to each other (see Milani et al. ${ }^{55,71}$ ). According to the ECC model, the normal mode associated with the most intense Raman line has been named the "ECC mode" and it has found applications in the interpretation of the Raman spectra of many different $\pi$-conjugated oligomers and polymers. Unlike oligomers and polymers, as well as the forms of carbon (e.g., graphite, diamond, fullerenes, and nanotubes), Raman analysis of sp-carbon chains has been investigated only recently in detail. Since polyynes/cumulenes are $\pi$-conjugated systems, the concepts developed in the AMT or the ECC model can be applied successfully. For infinite polyyne, the LO phonon at $q=0$ can be regarded as a stationary wave in which the CC bonds $r_{1}$ and $r_{2}$ simultaneously oscillate out-of-phase in the entire crystal. Hence the nuclear displacements associated with the LO phonon can be regarded as a collective BLA oscillation corresponding to the ECC mode. The frequency associated with this phonon can be analytically determined in terms of bond stretching force constants,,$^{54,55,57,76}$ effectively demonstrating the role of long-range interactions in determining the marked frequency decrease in the phonon dispersion (at $q=0$ ) as BLA approaches zero. This calculation can be conveniently carried out by adopting the infinite chain model with a unit cell of length $c$ containing two carbon atoms and by following Wilson's GF method ${ }^{72}$ generalized to periodic systems:

$$
G(\theta) F(\theta) L(\theta)=L(\theta) \Lambda(\theta)
$$

where $\theta=q c, G$ is the kinetic energy matrix, $F$ collects the valence force constants, $L$ describes the normal mode displacements along the internal coordinates (e.g., CC stretchings) and $\Lambda$ is the diagonal matrix that collects the phonon frequencies $\left(\lambda_{i}=\omega_{i}^{2}\right)$ at a given $\theta$ (the index $i$ labels the different phonon branches). The following solution is obtained for the LO phonons in correspondence to $q=0:^{54,55,71}$

$$
\omega^{2}=\frac{4 F_{\mathrm{R}}}{m} ; \quad F_{\mathrm{R}}=\frac{k_{1}+k_{2}}{2}+\sum_{n \geq 1}\left[f_{1}^{n}+f_{2}^{n}-2 f_{12}^{n}\right]
$$

In eqn (2), $F_{\mathrm{R}}$ is defined on the basis of several terms: $k_{1}$ and $k_{2}$ are the diagonal stretching force constants relative to the two bonds $\left(r_{1}, r_{2}\right)$ within the cell, and the various $f^{n}$ terms describe interaction stretching force constants at increasing distances $(n)$ along the chain. The variables $f_{1}^{n}$ and $f_{2}^{n}$ are associated with the interactions between the equivalent bonds belonging to different cells; $f_{12}^{n}$ describes the interaction between the non-equivalent bonds at the distance $n$. Eqn (2) demonstrates that the $\pi$-conjugation effects in CAWs are qualitatively similar to those occurring in other $\pi$-conjugated systems. For instance, it has been verified that the sequence of the interaction stretching force constants involving a given CC bond and a sequence of bonds along a path made by consecutive CC bonds has alternate signs in polyynes, ${ }^{55,62,73}$ similarly to polyenes and graphene..$^{52,67-69,74}$ As a consequence, the sum in eqn (2) has a negative value. This provides a $\pi$-conjugationdependent contribution that lowers the phonon frequency with respect to the value given by the diagonal force constants $k_{1}, k_{2}$. This decrease of $F_{\mathrm{R}}$ for increasing $\pi$-conjugation is responsible for the frequency dispersion of the intense Raman line associated with the ECC mode, which matches the behaviour at $q=0$ shown in Fig. 7a.

The physical origin of this phenomenon is the electronphonon coupling typical of these systems, even in the presence of $\pi$-electron delocalization. A simple model based on Hückel theory (i.e., tight binding restricted to $\pi$-electrons) can be introduced to better quantify these effects, ${ }^{55,62,71}$ leading to the following formulation of the matrix elements of the $F$ matrix of CC stretching force constants:

$$
F_{i j}=\delta_{i j} k_{i}^{\sigma}+4\left(\frac{\partial \beta}{\partial r}\right)^{2} \Pi_{i j}
$$

$\Pi_{i j}$ are the bond-bond polarizabilities and $\partial \beta / \partial r$ is the electron-phonon parameter, with $\beta$ the hopping integral of Hückel theory. This model allows for relating the electronic and vibrational properties, predicting the observed modulation of 


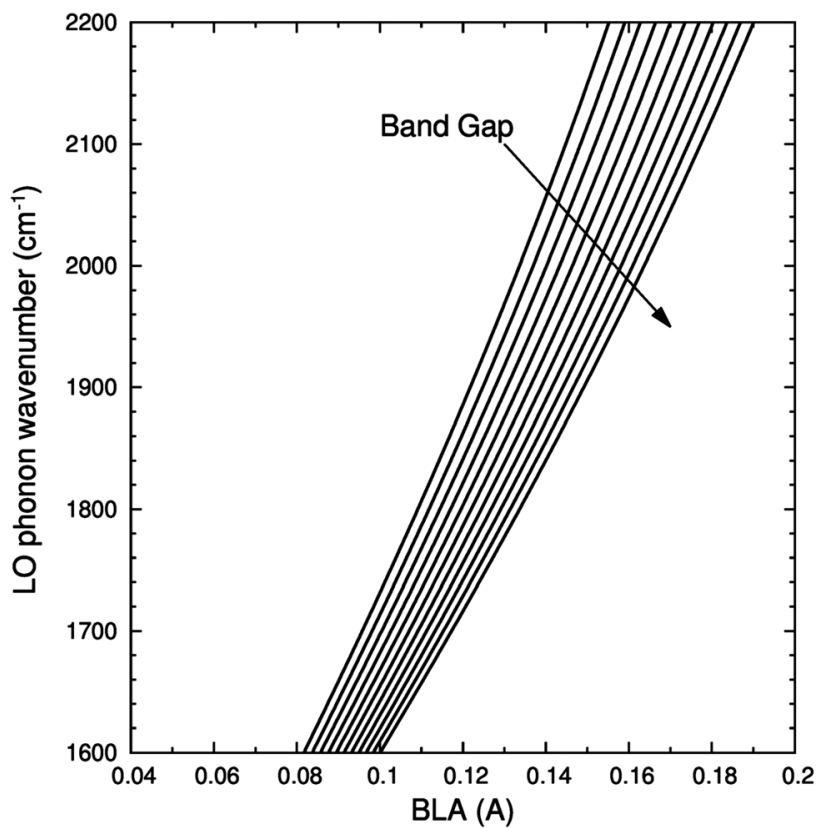

Fig. 8 Correlation between the LO phonon wavenumber (ECC mode) and bond length alternation (BLA) for different values of the electronic band gap, as obtained on the basis of Hückel theory applied to the linear infinite carbon chain. ${ }^{55,71}$

the electronic bands and phonon dispersion branches (Fig. 8), also in good agreement with quantum chemical calculations. ${ }^{53}$ The model also allows for introduction of the correlation that exists among the BLA, LO phonon wavenumber, and band gap in CAWs, which formally defines the connection between structural, electronic, and vibrational properties. ${ }^{71}$

The infinite chain model provides a reliable interpretation of the experimental data and captures the main trends observed for the electronic and vibrational properties, thus offering a useful unified framework for the understanding of real systems.

However, the use of periodic boundary conditions along the chain axis has a non-negligible influence on the spectroscopic selection rules: deviations of the chain structure from linearity, ${ }^{75}$ endgroup effects, ${ }^{61,76}$ and Peierls distortion (cumulene vs. polyyne) in long $\mathrm{C}_{n}$ wires require relaxing the infinite chain model, as described in the next section.

\subsection{Finite length sp-carbon systems: BLA and $\pi$-electron delocalization}

Moving from ideal (i.e., infinite chain) to real (i.e., finite length) structures, the endgroup effects play a leading role: the chemical nature of the endgroups influences the overall structure of the chain, increasing or decreasing BLA and, thus, modulating the energy gap and vibrational properties of the chain. The possibility of actually tuning the electronic properties and the conducting character by proper functionalization could be the key point for future technological applications, such as the realization of nanoscale cables and devices that have been proposed in theoretical studies. ${ }^{9-11,77}$
CAWs of increasing length show a decreasing BLA as a result of increasing $\pi$-conjugation..$^{54,73,78-81}$ However, the BLA $=0$ limit (cumulene) is not reached due to Peierls distortion. In this framework, the alternate (polyyne-like) structure found in most of the synthesized sp-carbon wires ${ }^{56}$ has been usually interpreted as the result of Peierls distortion, as also reported for other classes of $\pi$-conjugated materials. However, such an interpretation is prone to some criticism due to the fact that Peierls distortion is an effect that can be defined just for the infinite chain, not for relatively short CAWs for which the end functional groups are certainly the stronger agents in determining BLA (see below).

A detailed computational analysis on long uncapped cumulene-like $\mathrm{C}_{n}$ wires clearly demonstrates that Peierls distortion becomes effective only in very long chains $(n>52)$, where it can overcome the BLA decrease driven by $\pi$-electron delocalization. ${ }^{81}$ As a result of the balance between $\pi$-electron delocalization and Peierls distortion, $\mathrm{C}_{n}$ wires possess a cumulene-like structure (determined by the endgroup effects) for $n<52$. Longer $\mathrm{C}_{n}$ wires exhibit an alternated, polyyne-like structure due to the onset of Peierls distortion. This provides evidence that Peierls distortion may be rigorously defined just for an infinite chain and might not effectively explain the molecular structure observed in short CAWs. For these wires the presence of endgroup effects is essential ${ }^{80,81}$ and the carbon chain structure is mainly determined by the chemical nature of the endgroups, as highlighted by the values of BLA and CC bond lengths reported in Fig. 9 for a few series of sp-carbon molecules. ${ }^{61}$ In hydrogen endcapped wires, the $\mathrm{CH}$ single bonds require the presence of a triple bond on the adjacent CC linkage, so that a single bond is formed on the next $\mathrm{CC}$ and so on, thus inducing a polyyne-like structure. However, a vinylidene endgroup (i.e., $=\mathrm{CH}_{2}$ ) induces CC double bonds at the termini of the sp-chain, resulting in a more equalized cumulene-like structure.

Hence, for the same number of $\mathrm{C}$ atoms in the sp-chain, the vinylidene endgroup results in a substantially smaller BLA with respect to hydrogen endgroups. Clearly, this behaviour facilitates the design of new sp-carbon compounds with tunable properties by means of a proper choice of the endgroups and a synthetic approach. An example for this approach is represented by the recent work by Tykwinski et $a .^{82,83}$ where long cumulene-like CAWs (up to $9 \mathrm{C}=\mathrm{C}$ bonds) have been obtained by rational chemical synthesis. It should be noted that in finite-length wires, the cumulene-like geometry does not correspond to the ideal case with BLA = 0 . Finite cumulenes are systems showing small BLA values, which are markedly different from those found in polyynes. A further demonstration of the significance of finite length effects is given in Fig. 9d, which shows the bond lengths of quite long wires (30 carbon atoms) with different endgroups: CAWs still display very different CC bond lengths and BLA. Hence for these CAWs it is primarily the chemical nature of the end-caps that determines the structure, not the length of the chain. However, for fixed endgroups, BLA decreases with the chain length until it reaches an asymptotic lower limit, as generally expected by the increasing $\pi$-conjugation..$^{54,61,73,78-81,84}$ 

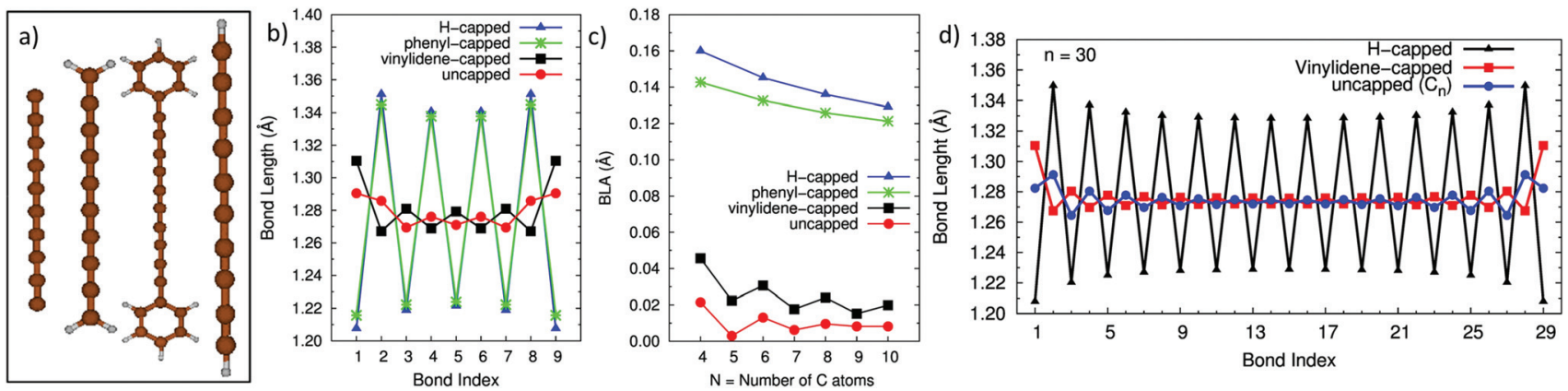

Fig. 9 (a) Structure of the considered wires (from left to right): uncapped, vinylidene-, phenyl- and H-endcapped; (b and c) bond length and bond length alternation (BLA) as a function of the number of carbon atoms for different types of wires. (d) Bond lengths in long wires (i.e., 30 carbon atoms) with different terminations. Data are from DFT calculations. Adapted with permission from ref. 8.

Based on the concepts illustrated above, a strict one-to-one correspondence between the infinite wire (i.e., carbyne) and finite-length CAWs cannot be established. However, due to the variance in BLA observed in CAWs as a function of length and/ or endgroups, finite CAWs should be related to different reference wire models possessing the same BLA as their finite counterpart. For instance, considering the electronic properties of finite CAWs, it becomes straightforward to suggest that by increasing the chain length (or by the proper choice of endgroups), the sp-chain will be characterized by smaller BLA values, leading to a smaller optical gap and a larger wavelength of the associated absorption band. This has been observed in many different CAWs, which also demonstrates the importance of spectroscopy as a quick and effective method for characterizing the structural properties of these systems. However, the absorption wavelengths observed in finite wires should be considered with care before extrapolating the electronic properties of the infinite polyyne. This is due to the fact that different finite-length CAWs are potentially associated with the parent systems with a different BLA. Extrapolation procedures have been discussed in some studies. ${ }^{36,85}$

\subsection{Finite length sp-carbon systems: molecular vibrations and Raman spectroscopy}

Parallel to the case of the electronic properties, where finite length CAWs can be placed in correspondence with an infinite chain model sharing the same BLA (see section 2.2), an analogous correlation should also be worked out for the vibrational properties of CAWs. The Raman spectra of hydrogen-endcapped polyynes show a similar pattern as polyenes, namely a very intense line that corresponds to the ECC mode, ${ }^{75,86}$ and this has also been named as " $\alpha$-line" in the literature. ${ }^{87} \mathrm{~A}$ second minor band ( $\beta$-line) is often observed, notably in shorter H-endcapped polyynes. ${ }^{54}$ These Raman signals fall in the $1800-2300 \mathrm{~cm}^{-1}$ spectral region. Through theoretical analysis and first-principles calculations it has been possible to assign the $\alpha$ - and $\beta$-lines to different collective stretching vibrations of CC bonds (i.e., BLA oscillation modes). ${ }^{87}$ This spectral region becomes specific for sp-carbon: no other carbon nanostructure shows any Raman signal in this region
(Fig. 10). In fact, the Raman spectra of $\mathrm{sp}^{2}$-based carbon nanostructures show a maximum frequency of about $1620 \mathrm{~cm}^{-1}$ that corresponds to the maximum in the phonon dispersion of graphite.

All the other features appearing above $2400 \mathrm{~cm}^{-1}$ are due to second order Raman scattering, including the 2D peak whose intensity and shape are peculiar in graphene. Similarly, the Raman peak of diamond at $1332 \mathrm{~cm}^{-1}$ represents the highest frequency in the phonon density of states. Hence, the Raman modes of CAWs appear in a spectral region where no other carbon systems show characteristic peaks.

Based on the results predicted for ideal carbyne ${ }^{54,55,62}$ and the general properties of polyconjugated molecules, the red shift of the most intense ECC Raman line observed when increasing the chain length has been qualitatively and quantitatively interpreted as a manifestation of large electronphonon coupling. With this aim, DFT calculations on finite length CAWs proved to be very helpful for spectroscopic characterization of these systems. ${ }^{55,59,61,73}$

The first interpretation of the Raman spectra of real spcarbon wires can be carried out by taking into account the infinite chain model which allows to interpret the $\alpha$ - and $\beta$-lines

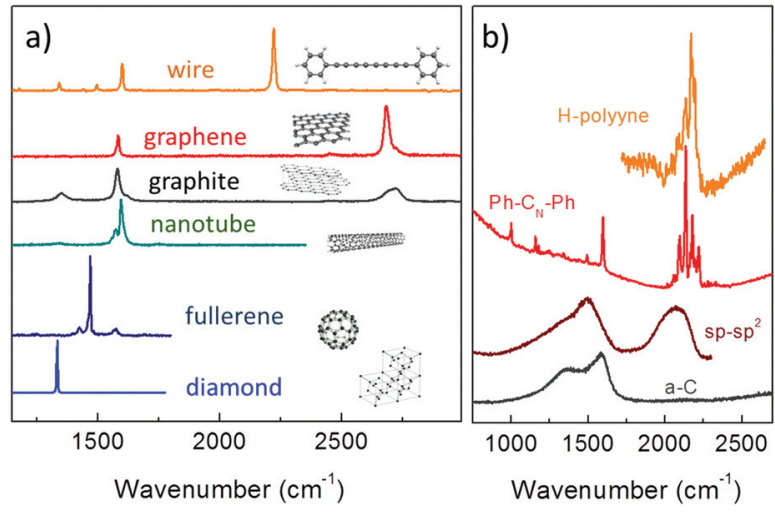

Fig. 10 (a) Experimental Raman spectra of carbon solids and (b) carbon-based nanostructures. 
on the basis of the LO phonon dispersion branches of carbyne, where the semiconductive-metallic transition (as BLA $\rightarrow 0$ ) involves the appearance of a Kohn anomaly at $\Gamma$ in the case of a cumulene-like chain. ${ }^{54,55,59,62,73}$

As mentioned above (section 2.2), collective CC stretching vibrations of CAWs involve stretching of the triple and single bonds, which give rise to Raman and IR signals in the range of $1800-2200 \mathrm{~cm}^{-1}$, depending on the length of the chain. ${ }^{28,75,76,87}$

In order to classify the CC stretching vibrations of the finite chains by establishing a correspondence with the LO phonon of the infinite polyyne, it is useful to adopt a procedure successfully employed in the past for the study of the vibrational dynamics of crystalline polymers. ${ }^{48,50}$ This procedure consists of selecting the suitable value of phonon wavevectors $(q)$ for which the vibrational wavenumbers of the oligomers can be placed as the discrete points on the phonon dispersion curves of the corresponding carbyne (with suitable BLA). It has been shown that these discrete $q$ values are given by the formula: ${ }^{48,73}$

$$
q_{j}=\frac{\pi}{c(N+1)} j ; \quad j=1,2,3, \ldots
$$

where $N$ is the number of unit cells which can be devised in the finite chain (oligomer). ${ }^{48,50}$ Provided that the endgroup effects have a limited influence on vibrational dynamics (which is often an accurate approximation for long enough oligomers), the procedure yields an accurate description of the normal modes which can be related to a given phonon branch.

In Fig. 11 this procedure has been applied to the longitudinal vibrations of the hydrogen-endcapped polyyne containing seven triple bonds. ${ }^{73}$ The vibrations of the molecule have been located on the (LO, LA) phonon dispersion of the infinite polyyne. The phonon dispersion was computed by using Wilson's approach (eqn (1) and (2)) and by considering the force field $F$ taken from the oligomer (i.e., considering the central triple bond of the finite chain as the reference 0th cell of the polymer and all interactions up to the edge of the molecule).

In non-conjugated polymers the oligomer-polymer correspondence can be established by considering just one oligomer long enough to minimize the endgroup effects. All longer oligomers can be related to this phonon dispersion. In contrast, each $\pi$-conjugated oligomer is characterized by a different degree of conjugation (hence different BLA). In principle, this implies that each oligomer possesses a different force field and leads to a different oligomer-derived phonon dispersion. From the experimental point of view, IR and Raman measurements carried out for a long super-trityl-endcapped polyyne have provided several CC stretching signals (Fig. 11). ${ }^{76}$ The assignment of these IR and Raman features by DFT calculations allows for drawing the corresponding phonon dispersion relationship. ${ }^{76}$ This shows that the relationships qualitatively follow theoretical expectations, ${ }^{54,73}$ and it exhibits the behaviour represented in Fig. 7 (left panel). In other words, the properties of finite-length CAWs can be related to
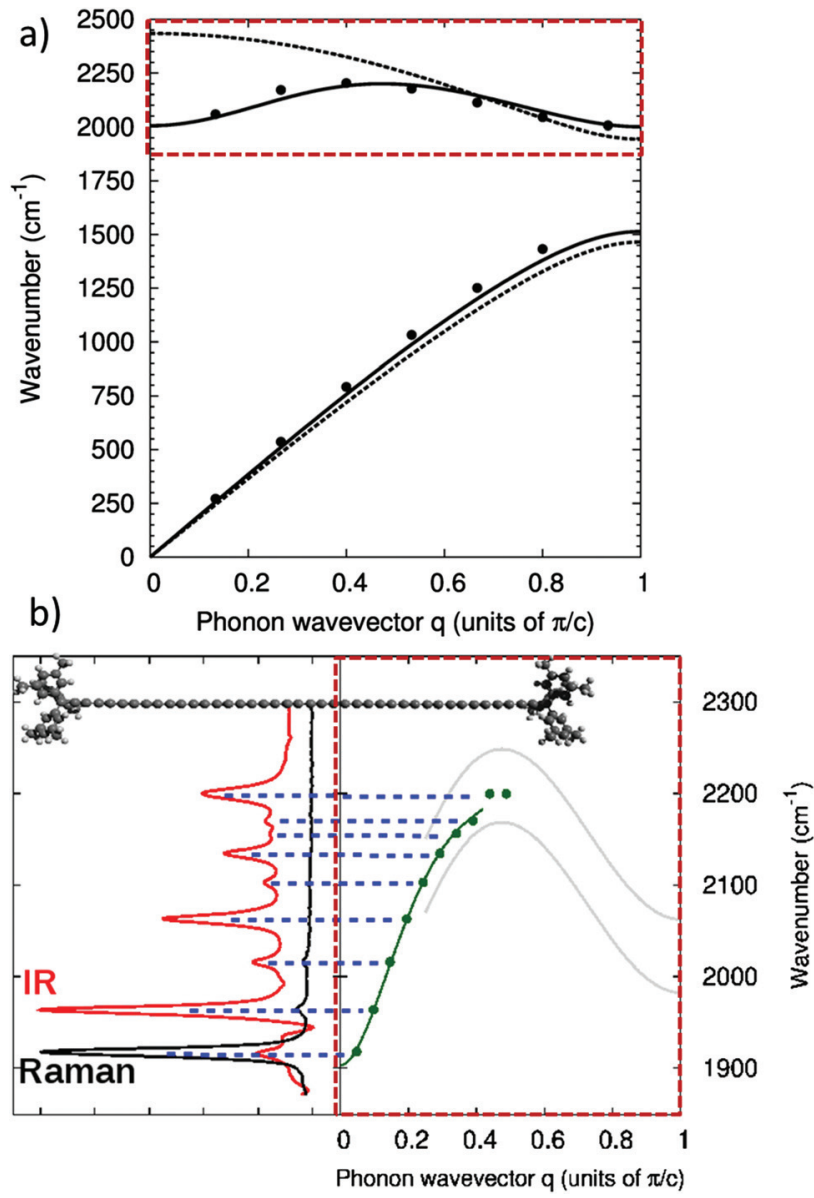

Fig. 11 (a) LO and LA phonon dispersion curves (full lines) obtained by using the DFT computed vibrational force field of a finite-length (7 triple bonds) hydrogen-endcapped polyyne. ${ }^{73}$ Black dots represent how the vibrational frequencies for this wire can be located on the phonon dispersion branches (see discussion in the text). For comparison, the phonon curves obtained by removing all long-range interactions ( $f_{n}$ terms in eqn (1)) are reported as dashed lines. Reprinted with permission from ref. 73, copyright 2007 American Chemical Society. (b) The same LO plot has been obtained on the basis of IR and Raman measurements carried out for a long polyyne endcapped with super-trityl groups (super-trityl $=$ tris (3,5-di-t-butylphenyl)methyl). ${ }^{76}$

that of the carbyne possessing the appropriate BLA for phonon dispersion branches. This model has been successfully adopted to give an interpretation of the Raman spectra of hydrogen-endcapped CAWs of increasing lengths ${ }^{54,73}$ and long polyynes (containing up to 20 conjugated triple bonds) endcapped with bulky groups. ${ }^{76}$

The strong Raman line (ECC mode) that is found on the LO dispersion corresponding to $j=1$ for long wires is essentially equivalent to the $q \approx 0$ phonon. ${ }^{54,73,76}$ In finite polyynes, IR modes are observed for the values $j>1$, and these can be simply considered as higher "harmonics" of the Raman active $j=1$ mode (see Fig. 11). ${ }^{75,76}$

The dependence of the vibrational properties on BLA makes the interpretation of the Raman spectra a non-trivial issue, requiring the support of numerical simulations. The 
chain conformation effects on the Raman spectrum have also been studied both experimentally and theoretically. ${ }^{75,76,79,88}$ These studies show that first-principles calculations can be helpful to elucidate the connections among structural, vibrational, and electronic properties of sp-carbon chains. Yang et $a l .{ }^{80}$ and Tommasini et al. ${ }^{73}$ have analysed the relationship between the BLA of hydrogen-endcapped polyynes and their Raman response, by means of DFT calculations.

The connection between the properties of finite-length CAWs and the ideal infinite polymers was characterized in different studies by using DFT calculations ${ }^{54,73}$ or tightbinding models, ${ }^{55,62,71,85}$ reconciling the approaches usually found in the Physics and Chemistry communities. The same methodologies were applied to the characterization of new synthetic series polyynes bearing suitable endgroups. ${ }^{75,89}$

The subtle problem of the existence of cumulene-like species and their detection by means of vibrational spectroscopy has been also addressed. ${ }^{61}$ This case is particularly interesting since it shows the inherent limits of the infinite chain model. In the literature, in fact, the possibility of observing cumulene-like wires by Raman spectroscopy has been contrasted by some authors based on the assumption that, as in model cumulene, they do not possess any optical phonon branch. However, Raman signatures of the existence of cumulene-like species have been suggested for mixed $\mathrm{sp} / \mathrm{sp}^{2}$-carbon nanostructures. ${ }^{27,88,90}$ This supposed controversy can be solved by relaxing the "carbyne perspective". Different from a model system (i.e., 1-D crystal) for which only phonons at $\Gamma$ could possess Raman activity, for finite wires also vibrations located on the LO branch at different points of the first Brillouin zone can be Raman active. This may happen as a result of endgroup effects, which constitute a perturbation over the strict periodic boundary conditions scheme introduced above. The Raman spectra of several cumulene-like wires $\left(\mathrm{C}_{n}\right)$ have been predicted by means of DFT calculations, ${ }^{61}$ and, interestingly, many vibrational transitions show a non-negligible Raman intensity, which demonstrates the possibility of detecting finite length cumulene-like wires by means of Raman spectroscopy. The reason for this behaviour lies in the interplay between the activation of out-of- $\Gamma$ normal modes and the molecular parameters governing the Raman activity. ${ }^{71}$ This originates in cumulenes a detectable Raman signal for LO modes other than ECC, as shown in Fig. 12. The Raman intensity of a given line is related to the square of the derivative of the polarizability tensor with respect to the associated normal mode $Q_{k}$.

This parameter can be written in terms of bond contributions as follows:

$$
\frac{\partial \alpha}{\partial Q_{k}}=\sum_{i} \frac{\partial \alpha}{\partial R_{i}} L_{i k}
$$

$\boldsymbol{L}_{k}$ is the eigenvector describing the normal mode $Q_{k}$ on the basis of internal valence coordinates (CC stretching, $R_{i}$ ) and $\partial \alpha / \partial R_{i}$ are the polarizability derivatives with respect to the individual CC stretching $\left(R_{i}\right)$ along the chain. ${ }^{91}$ In a

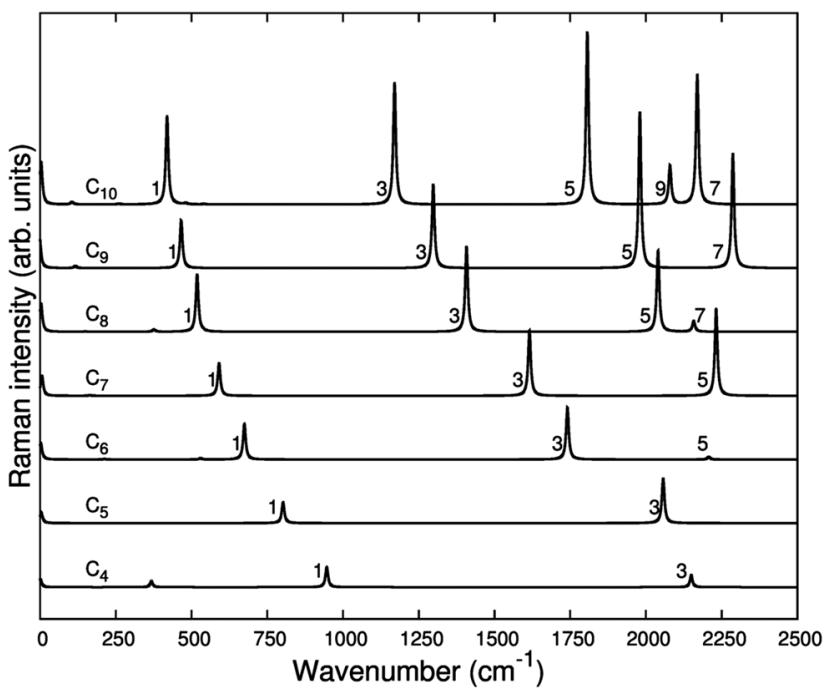

Fig. 12 Calculated Raman spectra of cumulene-like $C_{n}$ chains of different lengths. Bands are marked with labels indicating the number of nodes of the associated atomic displacements. Reprinted with permission ref. 61, copyright 2009 John Wiley and Sons.

cumulene, the $\partial \alpha / \partial R_{i}$ parameters are all very similar due to the similar character of all the CC bonds. The ECC mode describes the in-phase shrinking $\left(L_{i k}<0\right)$ and stretching $\left(L_{(i+1) k}>0\right)$ of the adjacent CC bonds $\left(R_{i}\right.$ and $\left.R_{i+1}\right)$ : therefore, almost equal $\partial \alpha / \partial R_{i}$ in the sum of eqn (5) are weighted by the $L_{i k}$ values of the opposite sign. This results in a vanishing sum, implying that the ECC mode has a small Raman intensity. This result is consistent with the infinite carbyne model. However, the infinite chain model cannot take into account the fact that other LO modes (out of $\Gamma$ ) have a different $L_{k}$ vector from that of the ECC mode.

This implies that the sum in eqn (5) can have a non-negligible value and the associated Raman lines may be detectable, as shown in Fig. 12 and described in ref. 61. Thus this discussion about cumulenes has a twofold meaning. On the one hand, it demonstrates the possibility of the spectroscopic detection of cumulene-like wires; on the other hand it reveals that in finite CAWs (real systems), some phenomena can be straightforwardly understood by only relaxing the infinite chain approximation. Based on the above discussion, it should be clear that the endgroup effects might influence the overall properties of sp-carbon wires, i.e., by modifying the molecular structure (BLA) that affects the electronic and vibrational properties. Therefore, by selecting the endgroups one can modulate the properties of the chain, which might be probed by spectroscopy; thanks to the evolution of the distinct marker bands observed in the Raman (or IR) spectra. In this context, Raman spectroscopy, supported by first-principles simulations, proves to be a powerful non-invasive characterization technique that can provide valuable information on the molecular properties of sp-carbon systems and can be useful in nanotechnology application scenarios. 
In section 4, we introduce a few recent case studies in which the vibrational spectroscopy proves to be particularly insightful to characterize sp-carbon systems with peculiar and not standard behaviour. For specific systems, we show that Raman spectroscopy allows for identification of CAWs with different lengths, and we highlight the observance of peculiar intra- and intermolecular effects.

\section{Synthesis and preparation of sp-carbon systems: a brief overview}

Today, many techniques can be found in the literature for the synthesis of sp-carbon systems. The different techniques are based on both physical and chemical strategies mainly in a bottom-up approach. ${ }^{22,92,93}$ Techniques based on physical vapour deposition methods depend on the production of a carbon vapour or plasma that is rapidly quenched to induce clustering of carbon atoms under out-of-equilibrium conditions. The carbon vapour can be produced by arc discharge or laser ablation, and quenching can be accomplished by the use of inert gas molecules or liquids (see Fig. 13). We mention supersonic cluster beam deposition (such as the pulsed microplasma cluster source - PMCS developed by P. Milani and coworkers), ${ }^{94}$ laser vaporization methods, ${ }^{95,96}$ and femtosecond laser ablation of graphite. ${ }^{97}$ These techniques typically produce solid samples in the form of thin films on substrates. The deposited material is usually composed of $\mathrm{sp}-\mathrm{sp}^{2}$ moieties where sp-wires are mixed in a mainly $\mathrm{sp}^{2}$-amorphous carbon matrix (sp-content up to $40 \%$ has been estimated in some cases). ${ }^{98-100}$

Laser ablation (with both fs and ns pulses) of solid carbon targets or suspensions (e.g., fullerenes and nanodiamond) produces solutions of isolated wires in the form of polyynes with

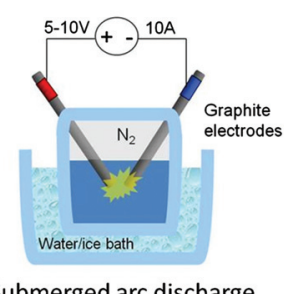

Submerged arc discharge

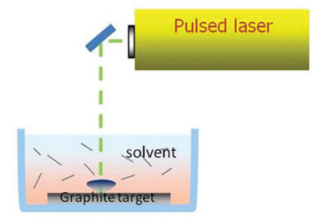

Pulsed laser ablation in liquid

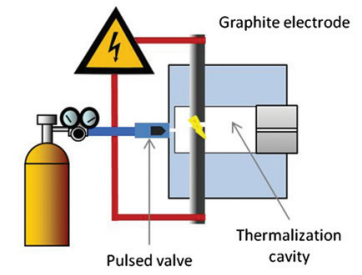

Pulsed Microplasma Cluster Source

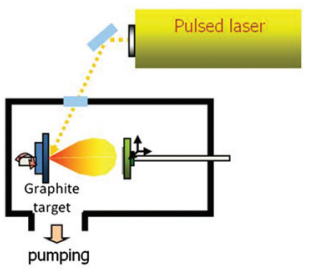

Pulsed laser deposition
Fig. 13 Sketches representing several known physical methods adapted to synthesize linear sp-carbon wires. Reprinted with permission from C.S. Casari Istituto Lombardo (Rend. Scienze), 2012, 146, 17-35 [eISSN 2384-986X]. an even number of carbon atoms. ${ }^{101-103}$ An easy-to-use and cost-effective technique for the synthesis of polyynes in solution is the arc discharge in liquids developed by F. Cataldo. ${ }^{103}$ This technique also allows one to control the chain termination by selecting suitable solvents. ${ }^{104}$

Chemical synthesis has been often used to synthesize systems in solution. Two approaches are often pursued. The first employs a polymerization strategy, as has been reviewed by Kudryavtsev. ${ }^{105,106}$ These syntheses are based on, for example, dehydropolycondensation of acetylene, polycondensation reactions of halides, and dehydrohalogenation of polymers, e.g., chemical carbonization of poly(vinylidene halides). In these cases, the resulting sp-carbon wires can be efficiently formed in a single step, but the products are polydisperse due to the nature of the synthetic protocol. Furthermore, solution state characterization of the resulting product is often challenging.

The second approach targets molecules with a defined length and endgroup constitution, typically via the dimerization reaction of ethynyl groups (Glaser reaction), ${ }^{107}$ with a defined length and specific endgroups, and the salient examples can be found in reviews by Szafert and Gladysz, ${ }^{93}$ Jevric and Nielsen, ${ }^{92}$ and Chalifoux and Tykwinski. ${ }^{108,109}$ In these cases, a homologous series of molecules can be made and studied as a function of length, allowing the prediction of properties of carbyne, as well as molecules that are not yet synthetically assessable.

Polyynes can also be formed on a surface, such as the formation of Pt-endcapped wires has been observed by TEM on graphene where Pt atoms act as nucleation sites of additional carbon atoms. ${ }^{110}$ TEM has been used to obtain wires suspended between graphene sheets in a top-down approach. The electron beam has been controlled to selectively remove carbon atoms from a graphene layer until a single atomic chain connecting two separate graphene edges is obtained and imaged. ${ }^{111}$ Other top-down methods are based on pulling a carbon nanotube or graphene in order to form a wire as predicted by theoretical studies.

Stability is a key issue in the synthesis of wires (Caution: the isolation of sp-carbon wires in the solid state should always be done with extreme care, since explosions are possible, especially in the case of $\mathrm{H}$-terminated polyynes). ${ }^{21}$ Crosslinking reactions of polyynes in the solid state play a lead role in reorganization processes of sp-wires towards amorphous $\mathrm{sp}^{2}$-carbon. To overcome this problem, one possibility is to stabilize the already formed wires. Some strategies of this kind have been proposed so far. H-Terminated polyynes produced by submerged arc discharge in water show reduced stability, ${ }^{86,112}$ while mixing the solution with silver colloids results in prolonged stability even in the solid state. In fact, by simply drying the solution one can obtain a solid sample (e.g., a thin film) consisting of an assembly of silver nanoparticles in which the wires display stability under ambient conditions for several weeks. ${ }^{113}$ A similar system of wires stabilized by silver can also be used to produce a nanocomposite, as shown by Hayashi and co-workers using poly(vinyl alcohol) as a 
polymeric matrix. ${ }^{114}$ An alternative strategy consists of designing and incorporating the endgroups at the termini of the wire during the synthesis, for example sterically bulky groups, in order to prevent intermolecular wire-wire interactions and subsequent cross-linking. Stable samples in powder form have been produced, ${ }^{115}$ as a chief example, Chalifoux and Tykwinski synthesized long wires (44 carbon atoms) stabilized by bulky endgroups. ${ }^{36}$

Polyynes are typically easier to produce than cumulenes probably due to higher stability, and nowadays polyynes up to 20 carbon atoms and more can be produced in solution even as size-selected samples. ${ }^{22,28,75}$ Step-wise syntheses offer the possibility of different terminating groups. ${ }^{93}$ On the other hand, cumulenes are more difficult to produce and there are far fewer reports on their synthesis and study in the literature. Cumulene-like structures have been observed together with

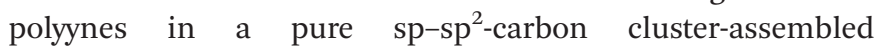
system. ${ }^{27,88}$ It has been observed that the cumulene fraction has a higher tendency to undergo transformation to $\mathrm{sp}^{2}$ carbon structures, confirming reduced stability toward oxygen exposure and high temperatures. ${ }^{98,116,117}$ Different terminations that can be provided by the amorphous carbon network can produce cumulene or polyyne structures, respectively. ${ }^{88}$ Short cumulenes can be synthesized in a similar way by controlling the termination-induced electronic arrangement. Such a strategy has been discussed in the review by Cadierno et al. ${ }^{118}$ and the synthesis of cumulene wires has been reported by Cataldo ${ }^{119}$ and more recently by Tykwinski and coworkers ${ }^{82,83}$ which describes cumulene wires up to 8 sp-carbon atoms produced by means of stepwise chemical synthesis. In all of these systems, the choice of terminal groups for the cumulene is fundamental for multiple reasons. For example, aromatic endgroups can strongly couple with the sp-chain, which then propagates through the wire structure. On the other hand, bulky endgroups are necessary in order to prevent intermolecular interactions among wires, and thus avoiding cross-linking reactions and conversion into amorphous $\mathrm{sp}^{2}$ carbon, as discussed also for polyynes.

\section{Raman, IR and SERS spectroscopy of carbon wires: recent investigations}

\subsection{Raman and SERS spectroscopy of carbon wires}

As mentioned above, the vibrational structure of CAWs depends on the length of the wire. This is important when dealing with the Raman active modes associated with CC stretching vibrations. DFT calculations of the Raman response of differently endcapped CAWs clearly show the marked dispersion of the strong Raman active mode associated with the BLA oscillation as a function of the number of sp-carbon atoms in the chain. ${ }^{7586,120}$ This sp-chain length dependence can be observed in Fig. 14 in the case of phenyl-endcapped polyynes.

The wavenumber of the characteristic Raman modes (BLA oscillation) sensibly decreases for increasing sp-chain lengths.

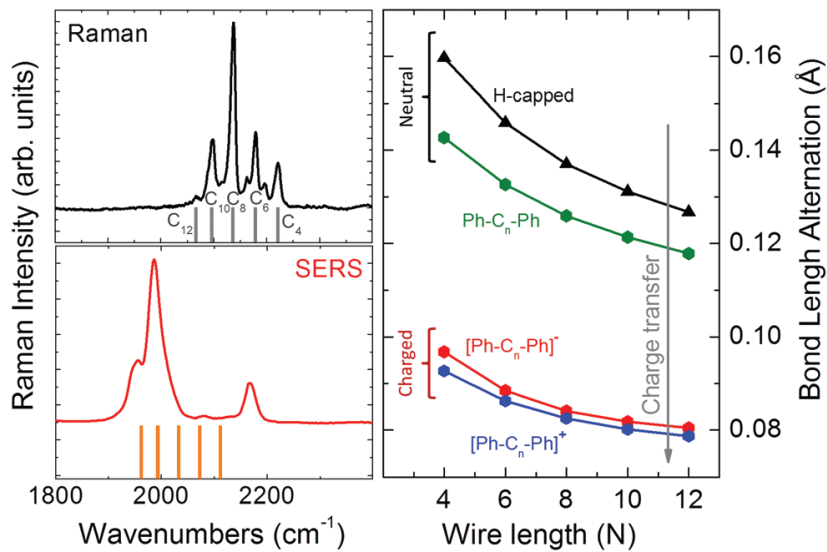

Fig. 14 Left panels: experimental Raman and SERS spectra (1064 nm) of phenyl-endcapped polyynes of silver nanoparticles, as the SERSactive medium. Lines show the theoretical position of peaks as a function of wire length according to DFT calculation of the Raman modes (PBE0/cc-pVTZ). ${ }^{79}$ Right panel: BLA values as a function of wire length for neutral and charged phenyl endcapped (Ph-endcapped) polyynes. BLA values of neutral $\mathrm{H}$-endcapped polyynes are also shown for comparison.

DFT calculations ${ }^{86}$ and experiments ${ }^{89}$ also show that the Raman intensity of these BLA-oscillation modes (ECC modes) promptly increases with the chain length, and it is sensitive to coupling with the $\pi$-conjugated caps, as it has been recently reported for cumulene-like systems. ${ }^{120}$ Although the correct Raman intensity behaviour as a function of chain length is not described very accurately by DFT methods, by summing up the contributions from each chain length (weighted by their abundance in the sample), one can obtain a reasonable representation of the experimental spectra in hydrogen-endcapped polyynes ${ }^{86}$ and phenyl-endcapped polyynes. $^{79}$ This approach allows for interpretation of the main Raman features of the spectra of CAW samples containing a distribution of chain lengths.

Amongst the wires investigated in detail by Raman spectroscopy, we mention polyynes terminated with hydrogen, metal atoms, phenyl and naphthalene groups, as well as systems with bulkier endgroups such as those synthesized by Tykwinski and co-workers. Hydrogen termination results in limited stability over time and the wires show transition towards $\mathrm{sp}^{2}$-carbon structures as a consequence of crosslinking reactions. ${ }^{112}$ Bulkier endgroups provide higher stability to CAWs. For example, phenyl-endcapped polyynes $\left(\mathrm{Ph}-\mathrm{C}_{n}-\mathrm{Ph}\right)$ up to $n=12$ are stable under ambient conditions even in a solid film when the solvent is completely removed. ${ }^{79}$ The Raman spectrum of samples of phenyl-endcapped polyynes with a distribution of chain lengths is characterized by the well-resolved peaks in the $2050-2250 \mathrm{~cm}^{-1}$ region. As depicted in Fig. 14, these features can be traced back to the distribution of the sp-chain lengths in the sample. DFT calculations of the Raman spectra of phenyl-endcapped polyynes of selected lengths show a significant red shift of the ECC mode for increasing chain lengths, consistent with the increase of $\pi$-conjugation. 
SERS is commonly employed to increase the sensitivity of Raman spectroscopy by many orders of magnitude sometimes reaching the single molecule level. It is based on the local enhancement of the electromagnetic field due to a resonance with surface plasmons present in metal nanoparticles. In addition to this enhancement (i.e., the electromagnetic SERS effect) there is also the possibility that the investigated system forms a complex with the metal nanoparticles due to charge transfer resulting in an additional enhancement that is usually called the chemical SERS effect. In the case of hydrogen endcapped polyynes, we observed an enhancement factor of $10^{6}$ with respect to normal Raman response. ${ }^{86}$ A peculiar effect is observed when hydrogen- and phenyl-endcapped polyynes interact with noble metal nanoparticles (i.e., silver and gold), such as those considered for SERS to increase the sensitivity of the Raman technique. ${ }^{79}$ We observe that the SERS spectrum is quite different from the Raman spectrum. A red-shift of the main Raman feature is accompanied by the appearance of new spectral features at lower wavenumbers (i.e., below $2000 \mathrm{~cm}^{-1}$ ), as shown in Fig. 14 for the case of diphenyl polyynes on silver nanoparticles. Similar spectral changes (i.e., from Raman to SERS) are also observed in the case of gold nanoparticles for different excitation wavelengths ranging from NIR to blue (1064-458 nm), suggesting that it is not a resonance-activated effect. $^{79}$

\subsection{Charge transfer effects in CAWs}

The spectral changes occurring upon interaction with metal nanoparticles, which are observed when comparing Raman with SERS (see above), suggest a chemical SERS effect with total enhancement factors up to $10^{6}$ (observed for hydrogenendcapped polyynes). ${ }^{86}$ A charge transfer between the metal and the carbon wire has been invoked to explain these remarkable spectral changes. ${ }^{79}$ In fact, by comparing ECC Raman frequencies calculated on neutral and charged CAWs, a relevant softening of the Raman modes and an increase of the intensity are observed for charged wires, as reported in Fig. 14. For instance, for a CAW of $n=8$ carbon atoms, a decrease of about $100 \mathrm{~cm}^{-1}$ upon charging the wire is predicted, both by adding or removing one electron. These theoretical predictions indicate that upon charge transfer, new Raman peaks would appear in the spectra at lower wavenumbers and with a higher Raman intensity, than those found for neutral wires. By evaluating the energy required for the formation of the two possible charged configurations, one can determine the direction of the charge transfer. The configuration with a positively charged metal and negatively charged wire becomes energetically favoured. Since charge transfer alters the electronic properties of the wire, effects on the structure are also expected, due to the characteristic and strong electron-phonon coupling that exists in $\pi$-conjugated systems. ${ }^{71}$ Charge transfer induces a decrease of BLA in the sp-carbon chain (Fig. 14), indicating that the wire evolves from an alternated (polyyne-like) to a more equalized (cumulene-like) wire configuration upon charge transfer. The reduction amounts to more than $30 \%$ for a singly charged wire and more than $60 \%$ for a doubly charged wire, reaching a lower value of $0.04 \AA$ for 12 carbon atoms. Recently synthesized cumulenes ${ }^{82}$ show an experimental BLA value as low as $0.038 \AA$ at the central bonds in the longest cumulene (formed by $9 \mathrm{C}=\mathrm{C}$ bonds). This compares well with the asymptotic BLA value of anionic phenyl-endcapped polyynes (Fig. 14) that can be thus considered to essentially possess a cumulene-like structure. Finally, it is important to remark that for finite-length wires, the ideal cumulene structure with $\mathrm{BLA}=0 \AA$ is not realistic due to the influence of the endgroups. The endgroup effects are stronger in shorter wires (see Fig. 9, where the BLA of finite cumulene-like systems is reported).

\subsection{Vibrational spectroscopy of excited states}

Another case demonstrating the existence of cumulene-like species can be found in ref. 117 where IR spectroscopy has been used to explain sp-carbon wires with a cumulene-like geometry induced by external effects, in particular the occurrence of photo-generated species trapped in a forbidden electronic state. In Fig. 15, IR spectra of a $\mathrm{C}_{12}$ polyyne after photo excitation are reported. After photo irradiation at a low temperature, a new band at about $2130 \mathrm{~cm}^{-1}$ appears in the IR spectrum, suggesting the photo generation of a new molecular species with particular vibrational and, hence, structural properties. Furthermore, by increasing the temperature, this band disappears, demonstrating that the process is reversible and should not be ascribed to any kind of degradation or transformation of the system. DFT calculations revealed that this feature can be ascribed to a molecule trapped in either the $S_{1}$ or $T_{1}$ excited

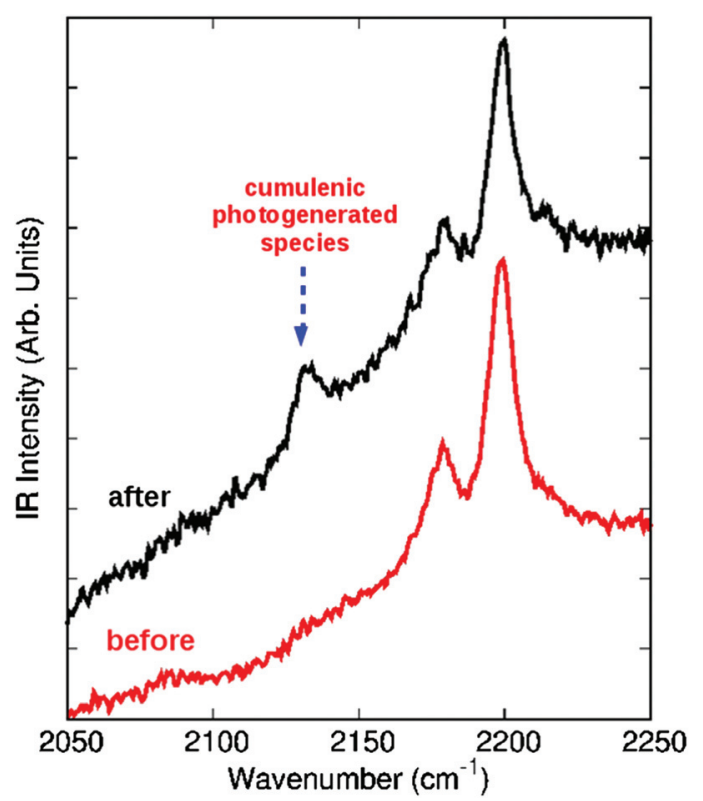

Fig. 15 IR spectra of adamantyl endcapped $\mathrm{C}_{12}$ polyyne embedded in a PMMA matrix: before and after UV irradiation with a $325 \mathrm{~nm}$ line recorded at $78 \mathrm{~K}$. The evolution of a new feature (associated with photo-generated cumulene-like species) is evident. Adapted with permission from ref. 117, copyright 2011 AIP Publishing LLC. 
state and which possesses a strong cumulene-like character with a very small value of BLA. ${ }^{117}$ These results demonstrate the possibility of observing and detecting cumulene-like CAWs and are among the first where the occurrence of these systems have been indeed established by means of experimental measurements. These experiments demonstrate the coupling between electronic and vibrational properties and verify the importance of investigating the excitation dynamics of sp-carbon wires, which are characterized by subtle and delicate phenomena involving different excited states. This latter point has been recently demonstrated by means of state-of-the art experimental techniques. ${ }^{121}$

\subsection{The peculiar Raman response of cumulene-like systems}

Long cumulenes have been recently synthesized by the Tykwinski group, ${ }^{82}$ thus offering opportunity to compare the molecular characteristics to the other sp-carbon wires. The cumulene-like systems are composed of 3, 5, 7 and 9 cumulated $\mathrm{C}=\mathrm{C}$ bonds, and they are endcapped with the aryl moieties (see Fig. 16). X-ray diffraction studies reveal that the BLA of these aryl endcapped cumulenes is substantially lower than that of a polyyne of a comparable chain length, which is also substantiated by DFT calculations. ${ }^{120}$ However, the structural data as a function of molecular length reveal a trend which appears to reach a limit before a cumulene-like structure with BLA $=0$ is achieved. ${ }^{82}$ This could indicate the onset of Peierls distortion, similarly to what has been reported by Kertesz on uncapped sp-carbon wires. ${ }^{84}$ Since the endgroups contribute to $\pi$-conjugation, a definitive explanation would require specific theoretical investigations, which are still lacking.

Raman spectroscopy ${ }^{120}$ of these long cumulenes has shown the direct implication of aryl groups to $\pi$-conjugation along the sp-chain through the unusually strong ECC Raman features, which are typical of bond-alternated (semiconductive) structures. Still, the observed low frequency of the ECC Raman signals indicates a system characterized by a low BLA (see Fig. 17). Compared to polyynes of a similar chain length and termination, ${ }^{76}$ cumulenes display a significantly less selective Raman signal, characterized by CC stretching lines attributed to the sp-chain (ECC) and to the endgroups (Fig. 17). At first glance, this different behaviour may be due to a decrease of
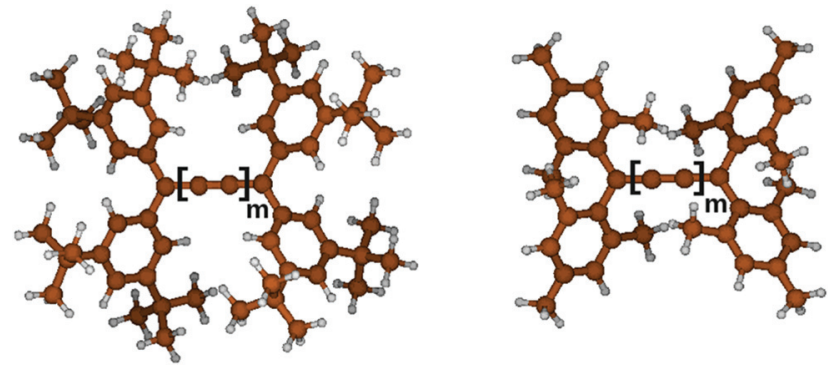

Fig. 16 Molecular structures of cumulenes: tBuPh[n] (left) and Mes[n] (right); $n=m+2$ indicates the number of cumulated double bonds in the carbon chain.

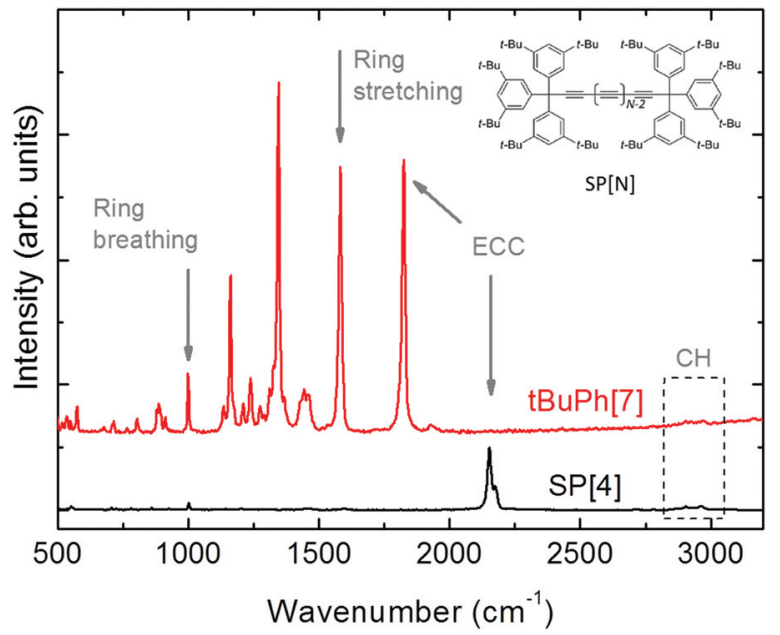

Fig. 17 Experimental FT-Raman spectra of polyyne SP[4] (black) and cumulene tBuPh[7], (red) normalized with respect to the aliphatic $\mathrm{CH}$ stretching region. The structure of polyyne SP[N] is shown in the inset, and the structure of $\mathrm{tBuPh}[n]$ is shown in Fig. 16. Adapted with permission from ref. 120, copyright 2014, American Chemical Society.

the Raman intensity of ECC modes in cumulenes compared to the endgroup modes, in contrast to the behaviour of polyynes for which ECC modes are strong and dominate over endgroup modes. ${ }^{89}$

However, a closer comparison of experimental data normalized with respect to the weak $\mathrm{CH}$ stretching signals (Fig. 17) reveals that the ECC signal of cumulene $\mathbf{t B u P h}[7]$ is comparable to that of a polyyne with a similar chain length, SP[4]. However, the phenyl modes of the cumulene are significantly stronger than those of the polyyne, which are barely observed as shown in Fig. 17.

DFT calculations reveal that the frontier orbitals of cumulenes, as expected, are delocalized over the sp-chain, but also significantly involve the aryl moieties of the endgroups. This behaviour is not observed in adamantyl endcapped polyynes $\operatorname{Ad}[\boldsymbol{n}]$ for which, in fact, we do not observe strong Raman modes of the endgroups compared to the modes of the wire. This interesting interplay of aryl groups with the cumulene chain may offer opportunities for fine-tuning of the molecular properties of cumulenes by proper chemical functionalization.

\section{Potential applications, novel structures and future perspectives}

As described in the previous sections, the structural tunability of CAWs implies that mechanical, electronic, and optical properties can be modulated to a wide extent, thus making these systems quite appealing for applications. Some efforts aimed at investigating the characteristics of sp-carbon systems have adopted advanced pump-probe techniques ${ }^{121}$ in order to characterize the dynamical evolution of electronic states and electrical measurements to assess the transport properties. 
The unusual behaviour of CAWs still deserves complete rationalization and will surely represent a fertile ground for future investigations.

\subsection{Optical and mechanical properties}

CAWs should be ideal systems to investigate the nonlinear optical (NLO) properties of 1-D conjugated systems. This hypothesis was first explored by two different methods. First, the non-resonant molecular second hyperpolarizabilities $\left(\gamma_{\text {ele }}\right)$ for the CAWs endcapped with silyl groups (TIPS $[\boldsymbol{n}]$ ) were determined using the differential optical Kerr effect (DOKE), ${ }^{122}$ and the $\gamma$-values show a power law increase as a function of length (n) (Fig. 18). ${ }^{123,124}$ This power law behaviour was stronger than that theoretically predicted for polyynes, and it was higher than that reported for polyenes and polyenynes. The second method evaluated the NLO behaviour based on the vibrational contribution to the second hyperpolarizability $\left(\gamma_{\mathrm{vib}}\right)$ for adamantyl endcapped CAWs $(\operatorname{Ad}[\boldsymbol{n}])$, using the quantitative Raman spectra and the Raman scattering intensities that correspond to the polyyne. ${ }^{125,126}$ It was quite remarkable that the $\gamma_{\text {vib }}$ values showed similar behaviour with respect to the chain length. ${ }^{89}$ It has been shown that the nature of the endgroups is negligible for longer CAWs in the two series based on UV/vis spectroscopy, ${ }^{127}$ and it was thus reasonable to conclude that the origin of the NLO behaviour in both the cases was derived primarily from the sp-hybridized carbon chain. ${ }^{128}$

The combined linear and nonlinear optical results from these studies implied that polyynes might function as ideal conjugated systems, ${ }^{129}$ which have motivated other studies of the NLO behaviour of sp-carbon containing systems, ${ }^{130,131}$ including optical limiting applications. ${ }^{132-136}$

According to their structure and bond coordination, CAWs can be expected to show very good mechanical properties. spHybridization should ensure an extremely high tensile strength that may outperform nanotubes and graphene. In

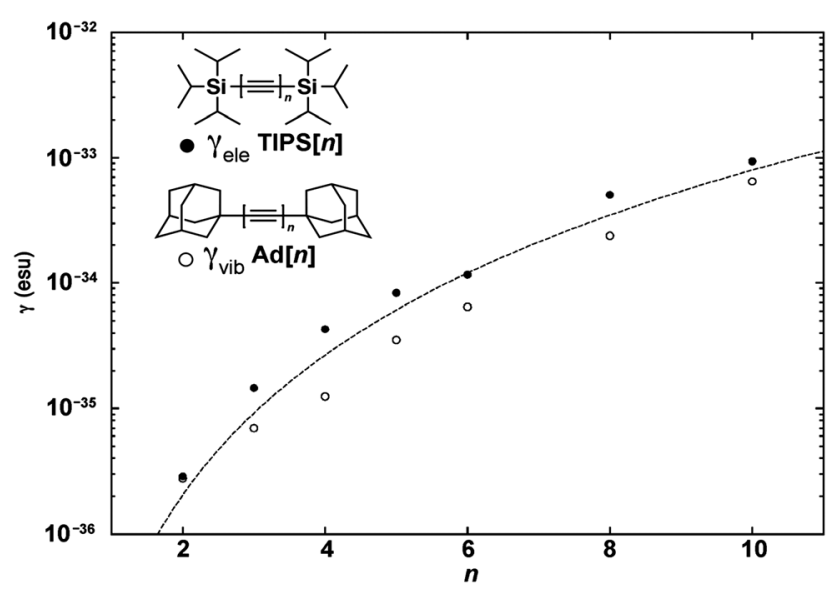

Fig. 18 Experimental $\gamma_{\text {ele }}(\bullet)$ and electronic $\gamma_{\text {vib }}(O)$ values for TIPS[ $\left.n\right]$ and $\mathrm{Ad}[n]$ polyynes, respectively, as a function of chain length (semi-log scale). The dashed line is a power-law fit to both datasets and it is meant only as a guide to the eye. recent years some papers have focused on theoretical calculations of mechanical properties of these systems, as 1-D wires with extraordinary properties. ${ }^{137,138}$

\subsection{Transport properties}

The interest in CAWs as conducting or functional wires comes from a wealth of theoretical studies pointing out the unique electronic and conductance properties of isolated systems. The pioneering theoretical investigations by Lang and Avouris showed an oscillatory behaviour with the number of carbon atoms of the conductance properties of wires contacted by gold leads. ${ }^{9,10}$ In particular, even-numbered wires are predicted to display lower conductance than odd-numbered wires. This is related to the density of states (DOS) behaviour at the Fermi level that is higher for even-numbered wires due to different populations of the HOMO level and the mixing with the metal states of the electrodes. Odd-numbered CAWs show constant conductance behaviour with the wire length. The conductivity values are close to the theoretical maximum of $2 G_{0}$, where $G_{0}=2 e^{2} / h=77 \mu \mathrm{S}$ (equivalent to a resistance of $12.9 \mathrm{k} \Omega$ ) is the quantum of conductance, according to the Landauer formula. ${ }^{139}$ For even-numbered wires the conductance increases with the number of carbon atoms indicating that the perturbation of the metal leads is more pronounced in short systems. A relevant charge transfer from the metal to the chain is considered to play an effective role in the conductance as a sort of doping effect that adds electrons to the partially filled HOMO level in even-numbered wires, while it contributes to the LUMO of odd-numbered wires that have a completely filled HOMO level. ${ }^{9,140}$

Larade et al. have predicted a transport regime characterized by negative differential resistance for CAWs connected to Al electrodes. ${ }^{140}$ The effect of strain and doping on the transport properties of infinite cumulene wires has been addressed by Tongay et $a{ }^{11}{ }^{11}$ Considering an electrode-wire-electrode configuration where metallic CAWs are used as electrodes, unstrained wires show quantum-ballistic transport with a constant conductance of $2 G_{0}$ for electron energy in a wide range around the Fermi level, while the application of a strain produces the appearance of an oscillatory behaviour of the conductance values as a function of electron energy. Yakobson and co-workers have investigated the bandgap dependence on mechanical strain pointing out a strain induced metal-to-insulator transition. On the one hand, they show that in a free unstrained chain the zero-point vibrational energy overcomes the barrier for the Peierls transition allowing for the existence of a long cumulene chain with metallic character. On the other hand, they underline that strain can be used to modify such an effect to favour the polyyne form with insulating behaviour. $^{77,137}$

While polyyne-like wires are expected to display metalliclike conductance when chemisorbed on metals like gold, ${ }^{141}$ recently, graphene sheets or nanoribbons have also been considered as contact electrodes. ${ }^{142,143}$ In particular, graphenewire-graphene systems are appealing as a pure carbon-based model for nanoscale-devices in which the linear energy depen- 
dence of the conductance in graphene can lead to a unique behaviour. Zanolli et al. ${ }^{143}$ showed that wires connected to graphene edges with zigzag or armchair structures show magnetic properties and spin-dependent transport. In general, the magnetization is strongly related to the chain structure. No magnetization is observed in even-numbered wires while oddnumbered wires show a net magnetization due to the local presence of uncompensated charge. In any case, the occurrence of spin polarization appears to be an intrinsic property of the wire that does not depend on the magnetization of the contacts.

Only a few studies have reported on the experimental transport properties of CAWs. The reason can be mainly due to the difficulty to obtain CAWs with controlled properties. The con-

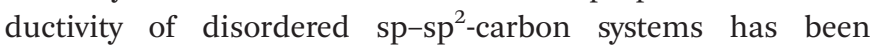
measured in situ as a function of the polyyne and cumulene content showing the contribution of sp-carbon to the electrical conductivity of the whole system. ${ }^{98}$ Moving to single molecule transport, Wang et al. firstly reported a measurement of the conduction of single CAWs by means of the STM-break junction method. ${ }^{144}$ Such a method is based on using the STM metal tip and a metal substrate as the two contacts of a molecular junction. By changing the tip-to-substrate distance, molecular junctions are repeatedly formed and broken. A plateau in the current-distance curve (at a given bias) indicates the molecular conductance. The same measurements can be performed by applying a mechanical strain that can induce the formation of a wire bridging two fragments (i.e., the so called mechanical-break junction method). A comparative study with both the techniques was conducted by Moreno-García et al. on wires with different lengths. ${ }^{145,146}$

The measured conductivity is much lower than the one predicted by ballistic conductance and this has been explained by the complex interaction between the terminations and the contact leads. Pt-endcapped polyynes have been explored experimentally in single molecule junctions. This work shows that vibronic features can be detected as satellites to the electronic transitions, which are assigned to longitudinal modes of the wires by theoretical studies. ${ }^{147}$

By shrinking a carbon nanotube under a TEM to induce narrowing, Yuzvinsky et al. measured conductance as a function of the tube diameter. Just before breaking, they observed a negative differential resistance, but no information on the nature of the connecting carbon chain was given. ${ }^{148}$ In this context, a detailed and systematic investigation of the conducting properties of CAW-based systems is an almost unexplored field. A few representative examples are reported in Fig. 19. A memory device based on forming and breaking wires by mechanical stress of graphene has been reported. ${ }^{149}$ Recently, F. Banhart and co-workers reported the measurements of transport properties in single CAWs suspended between graphene edges. ${ }^{7,150,151}$ The system was fabricated in a TEM starting from graphene and in situ characterized. In particular, by measuring the wires under strain they were able to observe a change in the current-voltage curves indicating a metal-to-semiconductor transition. ${ }^{151}$ Such experimental
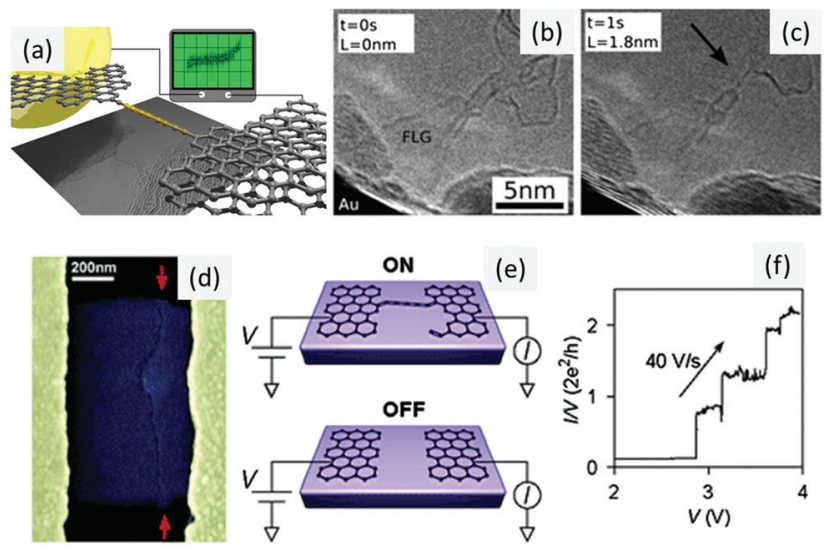

Fig. 19 Transport property measurement of single CAWs: (a-c) production by TEM technique and in situ measurement of wires suspended between graphene flakes (reprinted with permission from ref. 150, copyright 2013, American Chemical Society); (d-f) a graphene-based memristor device based on mechanically-induced formation and breaking of wires (reprinted with permission from ref. 149, copyright 2008, American Chemical Society).

results are in agreement with theoretical predictions, as discussed before.

\subsection{CAWs integrated with $\mathrm{sp}^{2}$-carbon}

Some possible systems where CAWs are integrated with $\mathrm{sp}^{2}$ carbon nanostructures are depicted in Fig. 20. The formation of an atomic junction between carbon nanotubes has been observed at the end of a shrinking procedure performed by mechanical pulling. ${ }^{138}$ CAWs have been observed in the core of carbon nanotubes (both single and multiple-walled), as evi-

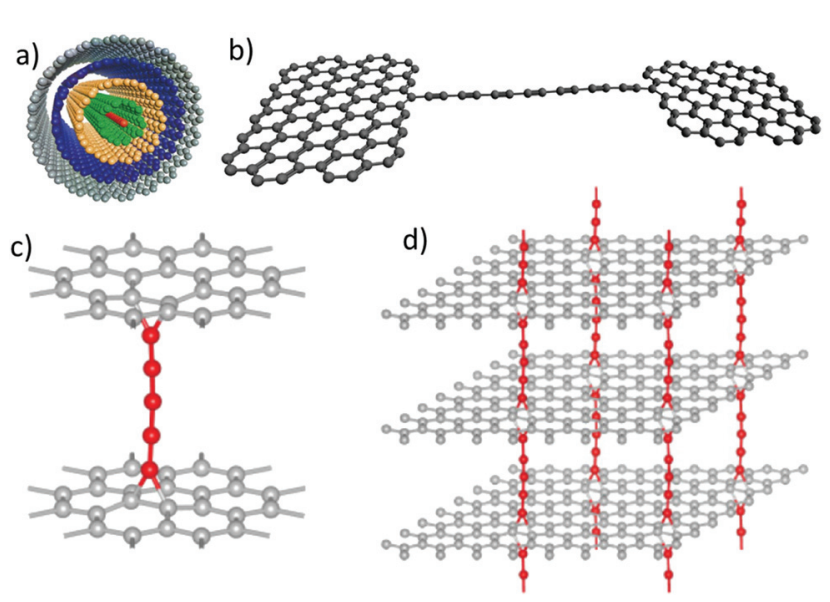

Fig. 20 Examples of carbon atomic wires integrated into $\mathrm{sp}^{2}$-carbon nanostructures: (a) CAW in the core of a multiwall carbon nanotube; (b) CAW suspended between two graphene edges; (c) CAW vertically grown from a graphene flake and (d) $\mathrm{sp}-\mathrm{sp}^{2}$-architecture comprising stacked graphene planes being separated by CAWs. Panel (a) is reprinted with permission from ref. 29, copyright 2003 by American Physical Society. Panels (c) and (d) are reprinted with permission from ref. 155, copyright 2011 by American Physical Society. 
denced by the TEM pictures of carbon nanotubes with regions showing apparently an odd number of walls one of which lies on the tube axis. ${ }^{29}$ The carbon nanotubes act as a protecting cage for CAWs thus permitting the observation of long wires, probably up to hundreds of carbon atoms. Hydrogen endcapped CAWs produced by laser ablation in liquids are able to fill the inner core of both multi- and single-walled carbon nanotubes. The tube protection allows for processing the system at a high temperature to promote the formation of long wires. ${ }^{152}$

Graphene has been considered as a natural termination for CAWs, either to have a pure carbon system with stable endgroups or as carbon-based electrodes for an ideal device setup. Graphene can induce a well-defined wire organization, depending on the type of bonding site provided for CAW anchoring, i.e., the $\mathrm{sp}^{2}$-sites are likely to promote cumulenetype while the $\mathrm{sp}^{3}$-sites will induce a polyyne-like organization. This can be used for the interpretation of the Raman spectrum

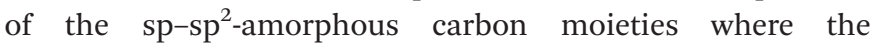
additional torsional and bending effects must be taken into account. ${ }^{88}$ A system built from two graphene edges connected by a CAW has been investigated by numerical simulations for unveiling the transport properties, as discussed above. Such an architecture is not only intriguing for theoretical predictions, but it has been recently realized and imaged by a TEMbased approach. ${ }^{7,31,111}$ CAWs between graphene domains have been realized but, even with TEM images showing the atomic structure of the chain, no evidence for either cumulene or polyyne structures was given. ${ }^{111}$ New insight into the structure of CAWs has been recently provided indicating a possible bond alternation in wires suspended between graphene edges. ${ }^{31}$ The possibility to produce CAWs by pulling a graphene flake was theoretically investigated showing that the formation of polyyne-like wires at the graphene edge is favoured by the presence of dimers and topological defects. ${ }^{153,154}$

The perpendicular growth of CAWs connecting the graphene sheets has been theoretically considered, toward producing carbon atomic pillars on graphene layers and even stacked layers being separated by wires of selected lengths (see Fig. 20). ${ }^{155}$ A bottom-up route to CAWs connected to graphene is represented by wires endcapped by coronene molecules as shown by numerical simulations. ${ }^{156}$ The computational investigation of CAWs bonded to moieties containing conjugated $\mathrm{sp}^{2}$-carbon atoms was carried out in the case of phenyl- and naphthyl-endcapped polyynes, ${ }^{79,157,158}$ demonstrating that these systems should show new chemical/physical phenomena deserving investigation.

The occurrence and structure of CAWs as the local defects of disordered carbon clusters were studied by simulating the rapid quenching of carbon vapours. ${ }^{100}$ On the other hand, the coagulation of CAWs in the $\mathrm{sp}-\mathrm{sp}^{2}$-networks has been observed as well. ${ }^{23,159}$

\section{$5.4 \mathrm{sp}-\mathrm{sp}^{2}$-hybrid architectures: graphynes and related novel structures}

A number of intriguing structures can be conceived by combining sp- and $\mathrm{sp}^{2}$-carbon atoms, including 2 -D crystals such as graphynes and graphdiynes (see e.g., the review by Ivanovskii). ${ }^{160}$ Such structures have been first outlined in 1987 by Baughman, Eckhardt and Kertesz. ${ }^{161}$ One simple example is given by using the linear $-\mathrm{C} \equiv \mathrm{C}$ - bonds to connect $\mathrm{sp}^{2}$-carbon hexagons thus producing the so-called $\gamma$-graphyne or 6,6,6-graphyne (see Fig. 21). Another possibility is to construct a honeycomb structure formed by the linear bonds only (both cumulene-like and polyyne-like), as a sp-carbon counterpart of graphene, called $\alpha$-graphyne or 18,18,18-graphyne. The nomenclature is based on three numbers indicating the number of carbon atoms in the smallest ring, in the next ring that is connected to the first one by a linear $-\mathrm{C} \equiv \mathrm{C}-$ bond, and in the ring that is in the ortho-position with respect to the previous two rings, respectively. According to this, $\alpha$-graphyne is $18,18,18$-graphyne while $\gamma$-graphyne is $6,6,6$-graphyne. The spcarbon content can vary from $75 \%$ in $\alpha$-graphyne, to $66.67 \%$ in 12,12,12-graphyne ( $\beta$-graphyne), 55\% for 6,6,12-graphyne, and down to $50 \%$ for $6,6,6$-graphyne.

Similar systems, graphdiynes, can be obtained by replacing a single acetylenic linkage with a double one (i.e., $-\mathrm{C} \equiv \mathrm{C}-$ $\mathrm{C} \equiv \mathrm{C}-$ ). The 6,6,6-graphdiyne structure has been reported by Haley et $a{ }^{162}$ as the most stable structure with diacetylenic units. According to theoretical predictions, such systems should show appealing electronic, optical, and mechanical properties. The presence of linear bonds should result in an increase of the Poisson's ratio and decrease of the in-plane stiffness with respect to graphene, while the bending stiffness is comparable. As in the case of single wires, graphyne shows a tunable gap when the system is under strain. The electronic properties have been computed for different structures showing the presence of Dirac's cones in the $K$ and $K^{\prime}$ points for 18,18,18graphyne, as it occurs in graphene. 12,12,12-Graphyne shows Dirac's cones in a low symmetry point along the $\Gamma M$ direction, as outlined by Görling and co-workers. ${ }^{163}$ They have also shown that Dirac's cones can occur in 2-D systems in the presence of hetero-atoms (e.g., $6_{\mathrm{BN}}, 6,12$ and $6\left(\mathrm{H}_{2}\right)$, 14,18-graphyne derivatives) and even with a rectangular structure, as the 6,6,12-graphyne, pointing out that a hexagonal symmetry (as

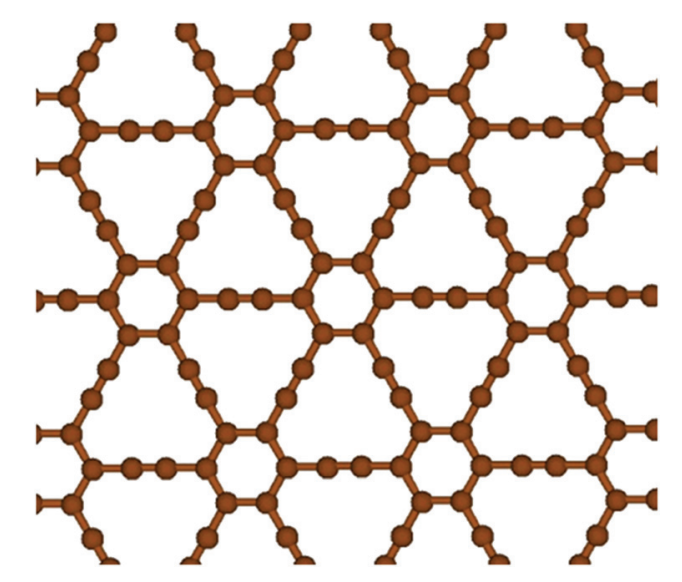

Fig. 21 Structure of $\gamma$-graphyne (6,6,6-graphyne). 
in the case of both 18,18,18- and 12,12,12-graphyne) is not required for the existence of the Dirac points (see Fig. 22). ${ }^{163,164}$ As a result of such peculiar electronic properties, a high charge carrier mobility has been predicted for these systems, comparable to graphene $\left(\mu=3 \times 10^{5} \mathrm{~cm}^{2} \mathrm{~V}^{-1} \mathrm{~s}^{-1}\right)$. 6,6,12-Graphyne should show a mobility value for both electrons and holes at least along one direction that could outperform graphene (i.e., about $5 \times 10^{5} \mathrm{~cm}^{2} \mathrm{~V}^{-1} \mathrm{~s}^{-1}$ for electrons). The vibrational properties have been calculated by Popov and Lambin for $\alpha$-, $\beta$-, and $\gamma$-graphynes to focus on Raman active modes and on the predicted Raman spectrum (see Fig. 23). ${ }^{165}$ Zone centre phonons below $1000 \mathrm{~cm}^{-1}$ are related to bending modes, and stretching modes appear at about $1000 \mathrm{~cm}^{-1}$ while stretching vibrations involving single and triple bonds are expected in the $2000-2200 \mathrm{~cm}^{-1}$ spectral region as in CAWs. All the Raman-active modes are in-plane. For example, in $\alpha$-graphyne the G-mode-like phonon is reduced to about $1000 \mathrm{~cm}^{-1}$ with a softening of about $50 \%$ with respect to the $\mathrm{G}$ mode in graphene or graphite $\left(1582 \mathrm{~cm}^{-1}\right)$.

Starting from graphyne and graphdiyne as the hybrid $\mathrm{sp}-\mathrm{sp}^{2}$-counterparts of graphene, a number of novel structures can be considered such as graphyne nanoribbons, nanotubes and fullerene-like cage structures (see e.g., review, ref. 160). For nanoribbons the energy gap increases with decreasing the ribbon width while nanotubes are expected to show metallic/ semiconducting behaviour depending on the chirality as for conventional $\mathrm{sp}^{2}$-carbon nanotubes. ${ }^{166-168}$

The theoretical results indicate great potential for such systems, however, experimental studies dealing with synthesis, characterization and application are still lacking. A study reported the synthesis of extended graphdiyne films even though there is no direct evidence for the ordered structure of

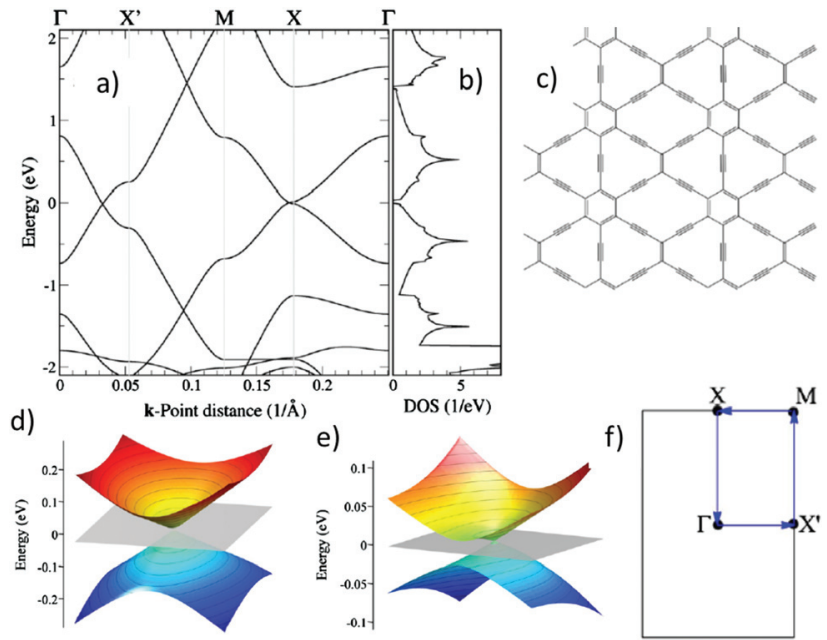

Fig. 22 (a) Electronic structure and (b) density of states of 6,6,12graphyne. The structure in the direct space is represented in (c). The two different Dirac's cones appearing along the $\Gamma M$ directions are represented in (d) and (e). The unit cell in the reciprocal space is schematized in (f). Reprinted with permission from ref. 163, copyright 2012 by American Physical Society.

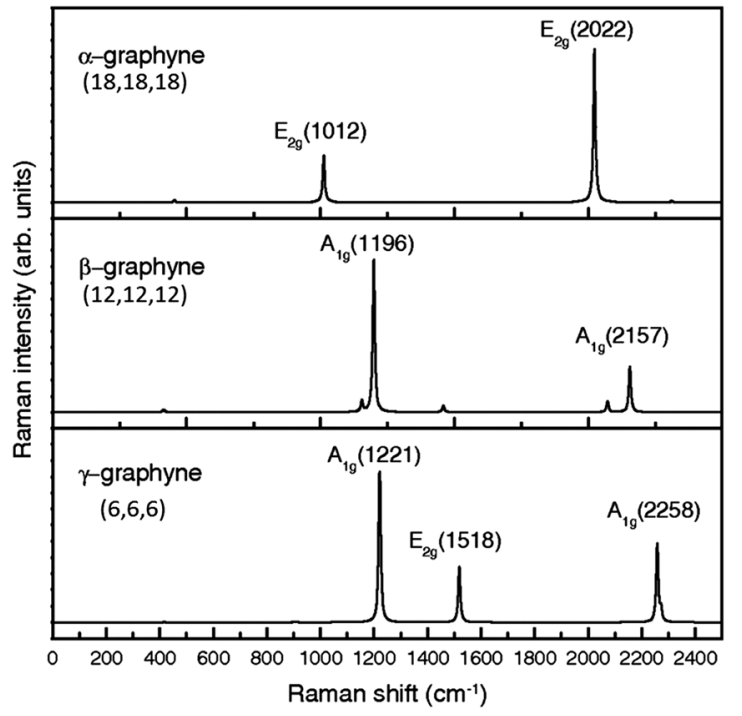

Fig. 23 Simulated Raman spectra of different types of graphyne systems. Reprinted with permission from ref. 165, copyright 2013 by American Physical Society.

the system. ${ }^{169}$ On the contrary, sp-sp ${ }^{2}$-carbon molecular building blocks for extended graphyne and graphdiynes have been produced by Diederich and co-workers, ${ }^{170,171}$ by Haley and coworkers ${ }^{162,172-174}$ and by other groups. ${ }^{175}$

Very recently conductivity measurements have been performed on model $\mathrm{sp}-\mathrm{sp}^{2}$-molecules (i.e., carbo-benzene and carbo- $n$-butadiene) at the single molecule level by means of the scanning tunnelling microscopy break junction method. ${ }^{176}$ Such systems can be considered as fundamental building blocks of extended $\alpha$-graphyne systems even though they have been endcapped with suitable anchoring groups to be connected with metal electrodes (i.e., the tip and the substrate). In the case of carbo-benzene, the results show single molecule conductance of about $100 \mathrm{nS}$ that is very high with respect to similar molecular fragments. For example, such a reported value is larger than a molecular fragment mimicking graphene (i.e., hexabenzocoronene) showing, with the same technique, a conductance of $14 \mathrm{nS}$ on a shorter distance (1.4 nm instead of $1.94 \mathrm{~nm}$ ). The reason for this performance is to be ascribed to the macro-aromatic ring that provides a rigid planar conformation, as supported by the comparison with other molecular fragments with a different degree of conformational rigidity. $^{176}$

A network of wires decorated by calcium atoms has been proposed as an efficient material for $\mathrm{H}$ storage, as a result of numerical calculations. ${ }^{177}$ Possible architectures comprise a wire-graphene system and a 3-D network of wires joined by $\mathrm{sp}^{3}$ links to form a diamond-like lattice.

While it is clear that theory predicts great potential for sp$\mathrm{sp}^{2}$-hybrids in nanotechnology applications, experimental results are obviously still needed. A few recent experiments confirm the peculiar properties of such systems at least at the level of molecular fragments, thus underlining that there is 
still a lot of work to be done to fill the large gap between theory and experiments.

\section{Conclusions and outlook}

In this review we have discussed sp-carbon nanostructures to underline the striking properties expected and, in some cases, observed in these systems. Carbon-atom wires are true 1-D systems with strong structure-property relationships that allow for tuning of the optical and electronic behaviour of these materials over a broad range of desired applications. Even though the existence of carbyne as the 'third carbon allotrope' is still controversial, sp-carbon nanostructures and molecules can now often be synthesized in stable forms and subsequently investigated. The structure and, hence, the functional properties of CAWs can be designed by controlling the wire length (i.e., number of carbon atoms) and endgroups. This opens the possibility to provide a new library of nanoscale systems with properties ranging from metallic through semiconducting to insulating. Such systems are appealing for many aspects of carbon science and have great potential in fields such as nano/molecular electronics and optoelectronics, as functional molecules, nanoscale structures and as assembled systems and molecular materials, just to name a few. In addition, great interest in the properties of graphene has driven the attention to hybrid $\mathrm{sp}-\mathrm{sp}^{2}$ architectures such as graphynes and graphdiynes. These 2-D materials and their molecular counterparts could show properties that might even be superior to graphene (e.g., charge carrier mobility, variable bandgap, multiple Dirac's cones, and behaviour as a topological insulator) as outlined by theoretical predictions. In this framework, there is also an obvious synergy between CAWs and graphene technologies, through the integration of spcarbon segments with graphene structures, such as wires bridging the graphene edges or suspended between graphene planes.

The seemingly limitless potential of sp-carbon-based nanoscale systems has been suggested in many theoretical studies, while we have only scraped the surface of discoveries in the experimental realm. There is, thus, a lot of work still to be done in order to unveil the potential of this "new" player in the vast family of carbon-based materials.

\section{Acknowledgements}

Funding in Erlangen from the Deutsche Forschungsgemeinschaft (DFG - SFB 953, "Synthetic Carbon Allotropes") is gratefully acknowledged.

\section{References}

1 A. Hirsch, Nat. Mater., 2010, 9, 868.

2 M. Dresselhaus, G. Dresselhaus and P. C. Eklund, Science of Fullerenes and Carbon Nanotubes, Academic Press, 1995.
3 A. H. Castro Neto, F. Guinea, N. M. R. Peres, K. S. Novoselov and A. K. Geim, Rev. Mod. Phys., 2009, 81, 109.

4 M. I. Knatsnelson, Graphene, Carbon in Two Dimesions, Cambridge Univ. Press, 2013.

5 A. C. Ferrari, F. Bonaccorso, V. Fal'ko, et al., Nanoscale, 2015, 7, 4598-4810.

6 H.-S. Philip Wong and D. Akinwande, Carbon Nanotube and Graphene Device Physics, Cambridge University Press, 2011.

7 F. Banhart, Beilstein J. Nanotechnol., 2015, 6, 559-569.

8 A. Milani, M. Tommasini, V. Russo, A. Li Bassi, A. Lucotti, F. Cataldo and C. S. Casari, Beilstein J. Nanotechnol., 2015, 6, 480-491.

9 N. D. Lang and Ph. Avouris, Phys. Rev. Lett., 2000, 84, 358.

10 N. Lang and P. Avouris, Phys. Rev. Lett., 1998, 81, 35153518.

11 S. Tongay, R. T. Senger, S. Dag and S. Ciraci, Phys. Rev. Lett., 2004, 93, 136404.

12 S. Tennant, Philos. Trans. R. Soc. London, A, 1797, 87, 123127.

13 R. B. Heimann, S. E. Evsyukov and L. Kavan, Carbyne and Carbynoid Structures, Kluwer Academic Publishers, 1999.

14 A. El Goresy and G. Donnay, Science, 1968, 161, 363.

15 A. G. Whittaker and P. L. Kintner, Science, 1969, 165, 589.

16 A. G. Whittaker, Science, 1978, 200, 763.

17 P. P. K. Smith and P. R. Buseck, Science, 1982, 216, 984.

18 A. G. Whittaker, Science, 1985, 229, 485.

19 P. P. K. Smith and P. R. Buseck, Science, 1985, 229, 486.

20 H. Kroto, Rev. Mod. Phys., 1997, 69, 703.

21 R. H. Baughman, Science, 1009, 312, 2006.

22 F. Cataldo, Polyynes: Synthesis, Properties, and Applications, Taylor \& Francis, 2005.

23 T. Wakabayashi, A. L. Ong, D. Strelnikov and W. Krätschmer, J. Phys. Chem. B, 2004, 108, 3686.

24 L. Ravagnan, F. Siviero, C. S. Casari, A. Li Bassi, C. Lenardi, C. E. Bottani and P. Milani, Carbon, 2005, 43, 1337-1339.

25 L. Ravagnan, T. Mazza, G. Bongiorno, M. Devetta, M. Amati, P. Milani, P. Piseri, M. Coreno, C. Lenardi, F. Evangelista and P. Rudolf, Chem. Commun., 2011, 47, 2952.

26 A. A. Zaidi, A. Hu, M. J. Wesolowski, X. Fu, J. H. Sanderson, Y. Zhou and W. W. Duley, Carbon, 2010, 48, 2517.

27 L. Ravagnan, F. Siviero, C. Lenardi, P. Piseri, E. Barborini, P. Milani, C. S. Casari, A. Li Bassi and C. E. Bottani, Phys. Rev. Lett., 2002, 89, 285506.

28 H. Tabata, M. Fujii and S. Hayashi, Carbon, 2006, 44, 522.

29 X. Zhao, Y. Ando, Y. Liu, M. Jinno and T. Suzuki, Phys. Rev. Lett., 2003, 90, 187401.

30 C. Jin, H. Lan, L. Peng, K. Suenaga and S. Iijima, Phys. Rev. Lett., 2009, 102, 205501.

31 G. Casillas, A. Mayoral, M. Liu, A. Ponce, V. I. Artyukhov, B. I. Yakobson and M. Jose-Yacaman, Carbon, 2014, 66, 436-441. 
32 Y. Wang, Y. Huang, B. Yang and R. Liu, Carbon, 2006, 44, 456.

33 E. Cazzanelli, M. Castriota, L. S. Caputi, A. Cupolillo, C. Giallombardo and L. Papagno, Phys. Rev. B: Condens. Matter, 2007, 75, 121405R.

34 D. Nishide, H. Dohi, T. Wakabayashi, E. Nishibori, S. Aoyagi, M. Ishida, S. Kikuchi, R. Kitaura, T. Sugai, M. Sakata and H. Shinohara, Chem. Phys. Lett., 2006, 428, 356.

35 D. Nishide, T. Wakabayashi, T. Sugai, R. Kitaura, H. Kataura, Y. Achiba and H. Shinohara, J. Phys. Chem. C, 2007, 111, 5178.

36 W. A. Chalifoux and R. R. Tykwinski, Nat. Chem., 2010, 2, 967-971.

37 A. Mendoza, Y. Ishihara and P. S. Baran, Nat. Chem., 2012, 4, 21-25.

38 R. O. Jones and G. Seifert, Phys. Rev. Lett., 1997, 79, 443.

39 M. Saito and Y. Okamoto, Phys. Rev. B: Condens. Matter, 1999, 60, 8939.

40 T. Torelli and L. Mitas, Phys. Rev. Lett., 2000, 85, 1702.

41 A. Abdurahman, A. Shukla and M. Dolg, Phys. Rev. B: Condens. Matter, 2002, 65, 115106.

42 A. Karpfen, J. Phys. C: Solid State Phys., 1979, 12, 3227.

43 M. Springborg, J. Phys. C: Solid State Phys., 1986, 19, 4473.

44 A. Rusznyak, V. Zolyomi, J. Kurti, S. Yang and M. Kertesz, Phys. Rev. B: Condens. Matter, 2005, 72, 155420.

45 U. Molder, P. Burk and I. A. Koppel, J. Mol. Struct., 2004, 712, 81.

46 M. Kertesz and S. Yang, Phys. Chem. Chem. Phys., 2009, 11, 425.

47 Y. Liu, R. O. Jones, X. Zhao and Y. Ando, Phys. Rev. B: Condens. Matter, 2003, 68, 125413.

48 R. Zbinden, Infrared Spectroscopy of High Polymers, Academic Press, 1964.

49 P. C. Painter, M. M. Coleman and J. L. Koenig, The Theory of Vibrational Spectroscopy and Its Application to Polymeric Materials, Wiley \& Sons, 1982.

50 G. Zerbi, in Advances in Infrared and Raman spectroscopy, ed. R. J. H. Clark and R. R. Hester, Wiley, 1984, vol. 11, p. 301 .

51 C. Castiglioni, in Vibrational Spectroscopy of Polymers: Principles and Practices, ed. N. J. Everall, J. M. Chalmers and P. R. Griffiths, Wiley, 2007, p. 455.

52 M. Del Zoppo, C. Castiglioni, P. Zuliani and G. Zerbi, in Handbook of Conductive Polymers, ed. T. Skotheim, R. L. Elsembaumer and J. Reynoilds, Dekker, 2nd edn, 1998, p. 765.

53 R. Hoffmann, Tetrahedron, 1966, 22, 521-538.

54 A. Milani, M. Tommasini, M. Del Zoppo, C. Castiglioni and G. Zerbi, Phys. Rev. B: Condens. Matter, 2006, 74, 153418.

55 A. Milani, M. Tommasini and G. Zerbi, J. Chem. Phys., 2008, 128, 064501.

56 W. A. Chalifoux, R. McDonald, M. J. Ferguson and R. R. Tykwinski, Angew. Chem., Int. Ed., 2009, 48, 7915-7919.
57 R. E. Peierls, Quantum Theory of Solids, Oxford University Press, 2001.

58 R. Hoffmann, Angew. Chem., Int. Ed. Engl., 1987, 26, 846878.

59 A. Milani, M. Tommasini, D. Fazzi, C. Castiglioni, M. Del Zoppo and G. Zerbi, J. Raman Spectrosc., 2008, 39, 164.

60 N. W. Ashcroft and N. D. Mermin, Solid State Physics, Saunders, 1976.

61 F. Innocenti, A. Milani and C. Castiglioni, J. Raman Spectrosc., 2010, 41, 226.

62 M. Tommasini, A. Milani, D. Fazzi, M. Del Zoppo, C. Castiglioni and G. Zerbi, Physica E, 2008, 40, 2570.

63 S. Piscanec, M. Lazzeri, F. Mauri, A. C. Ferrari and J. Robertson, Phys. Rev. Lett., 2004, 93, 185503.

64 M. Lazzeri, S. Piscanec, F. Mauri, A. C. Ferrari and J. Robertson, Phys. Rev. B: Condens. Matter, 2006, 73, 155426.

65 S. Piscanec, M. Lazzeri, J. Robertson, A. C. Ferrari and F. Mauri, Phys. Rev. B: Condens. Matter, 2006, 75, 035427.

66 E. Di Donato, M. Tommasini, C. Castiglioni and G. Zerbi, Phys. Rev. B: Condens. Matter, 2006, 74, 184306.

67 G. Zerbi, in Vibrational Spectroscopy of Polymers: Principles and Practice, ed. N. J. Everall, J. M. Chalmers and P. R. Griffiths, Wiley, 2007, p. 487.

68 C. Castiglioni, M. Gussoni, J. T. Lopez-Navarrete and G. Zerbi, Solid State Commun., 1988, 65, 625.

69 C. Castiglioni, M. Tommasini and G. Zerbi, Philos. Trans. R. Soc. London, Ser. A, 2004, 362, 2425.

70 E. Ehrenfreund, Z. Vardeny, O. Brafman and B. Horovitz, Phys. Rev. B: Condens. Matter, 1987, 36, 1535.

71 A. Milani, M. Tommasini and G. Zerbi, J. Raman Spectrosc., 2009, 40, 1931.

72 E. B. Wilson, J. C. Decius and P. C. Cross, Molecular Vibrations: The Theory of Infrared and Raman Vibrational Spectra, Dover, 1980.

73 M. Tommasini, D. Fazzi, A. Milani, M. Del Zoppo, C. Castiglioni and G. Zerbi, J. Phys. Chem. A, 2007, 111, 11645.

74 C. Mapelli, C. Castiglioni, G. Zerbi and K. Müllen, Phys. Rev. B: Condens. Matter, 1999, 60, 12710.

75 A. Lucotti, M. Tommasini, D. Fazzi, M. Del Zoppo, W. A. Chalifoux, M. J. Ferguson, G. Zerbi and R. R. Tykwinski, J. Am. Chem. Soc., 2009, 131, 4239.

76 N. R. Agarwal, A. Lucotti, D. Fazzi, M. Tommasini, C. Castiglioni, W. A. Chalifoux and R. R. Tykwinski, J. Raman Spectrosc., 2013, 44, 1398.

77 V. I. Artyukhov, M. Liu and B. I. Yakobson, Nano Lett., 2014, 14(8), 4224-4229.

78 M. Kertesz, C. Ho Choi and S. Yang, Chem. Rev., 2005, $105,3448$.

79 A. Milani, A. Lucotti, V. Russo, M. Tommasini, F. Cataldo, A. Li Bassi and C. S. Casari, J. Phys. Chem. C, 2011, 115, 12836.

80 S. Yang, M. Kertesz, V. Zolyomi and J. Kurti, J. Phys. Chem. A, 2007, 111, 2434.

81 S. Yang and M. Kertesz, J. Phys. Chem. A, 2008, 112, 146. 
82 J. A. Januszewski, D. Wendinger, C. D. Methfessel, F. Hampel and R. R. Tykwinski, Angew. Chem., Int. Ed., 2013, 52, 1817.

83 J. A. Januszewski and R. R. Tykwinski, Chem. Soc. Rev., 2014, 43, 3184.

84 S. Yang and M. Kertesz, J. Phys. Chem. A, 2006, 110, 9771.

85 M. Tommasini, D. Fazzi, A. Milani, M. Del Zoppo, C. Castiglioni and G. Zerbi, Chem. Phys. Lett., 2007, 450, 86.

86 A. Lucotti, M. Tommasini, M. Del Zoppo, C. Castiglioni, G. Zerbi, F. Cataldo, C. S. Casari, A. Li Bassi, V. Russo, M. Bogana and C. E. Bottani, Chem. Phys. Lett., 2006, 417, 78.

87 H. Tabata, M. Fujii, S. Hayashi, T. Doi and T. Wakabayashi, Carbon, 2006, 44, 3168.

88 L. Ravagnan, N. Manini, E. Cinquanta, G. Onida, D. Sangalli, C. Motta, M. Devetta, A. Bordoni, P. Piseri and P. Milani, Phys. Rev. Lett., 2009, 102, 245502.

89 A. Lucotti, M. Tommasini, D. Fazzi, M. Del Zoppo, W. A. Chalifoux, R. R. Tykwinski and G. Zerbi, J. Raman Spectrosc., 2012, 43, 1293.

90 C. S. Casari, A. Li Bassi, L. Ravagnan, F. Siviero, C. Lenardi, P. Piseri, G. Bongiorno, C. E. Bottani and P. Milani, Phys. Rev. B: Condens. Matter, 2004, 69, 075422.

91 D. A. Long, The Raman Effect: A Unified Treatment of the Theory of Raman Scattering by Molecules, John Wiley \& Sons, 2002.

92 M. Jevric and M. B. Nielsen, Asian J. Org. Chem., 2015, 4, 286.

93 S. Szafert and J. A. Gladysz, Chem. Rev., 2003, 103, 4175.

94 P. Milani and S. Iannotta, Cluster Beam Synthesis of Nanostructured Materials, Springer, 1999.

95 G. Compagnini, S. Battiato, O. Puglisi, G. A. Baratta and G. Strazzulla, Carbon, 2005, 43, 3025.

96 T. Wakabayashi, H. Nagayama, K. Daigoku, Y. Kiyooka and K. Hashimoto, Chem. Phys. Lett., 2007, 446, 65-70.

97 A. Hu, et al., J. Chem. Phys., 2007, 126, 154705.

98 L. Ravagnan, P. Piseri, M. Bruzzi, S. Miglio, G. Bongiorno, A. Baserga, C. S. Casari, A. Li Bassi, C. Lenardi, Y. Yamaguchi, T. Wakabayashi, C. E. Bottani and P. Milani, Phys. Rev. Lett., 2007, 98, 216103.

99 L. Ravagnan, G. Bongiorno, D. Bandiera, E. Salis, P. Piseri, P. Milani, C. Lenardi, M. Coreno, M. de Simone and K. C. Prince, Carbon, 2006, 44, 1518.

100 M. Bogana, L. Ravagnan, C. S. Casari, A. Zivelonghi, A. Baserga, A. Li Bassi, C. E. Bottani, S. Vinati, E. Salis, P. Piseri, E. Barborini, L. Colombo and P. Milani, New J. Phys., 2005, 7, 81.

101 H. Tabata, M. Fujii and S. Hayashi, Chem. Phys. Lett., 2006, 420, 166.

102 A. Hu, M. Rybachuk, Q.-B. Lu and W. W. Duley, Appl. Phys. Lett., 2007, 91, 131906.

103 F. Cataldo, Carbon, 2003, 41, 2671.

104 F. Cataldo, Tetrahedron, 2004, 60, 4265.

105 Y. P. Kudryavtsev, R. B. Heimann and S. E. Evsyukov, J. Mater. Sci., 1996, 31, 5557.
106 Y. P. Kudryavtsev, S. E. Evsyukov, M. B. Guseva, V. G. Babaev and V. V. Khvostov, Russ. Chem. Bull., 1993, 42, 399.

107 P. Siemsen, R. C. Livingston and F. Diederich, Angew. Chem., Int. Ed., 2000, 39, 2633.

108 W. A. Chalifoux and R. R. Tykwinski, C. R. Chim., 2009, $12,341$.

109 R. R. Tykwinski, Chem. Rec., 2015, DOI: 10.1002/ tcr.201500018.

110 E. Kano, M. Takeguchi, J. Fujita and A. Hashimoto, Carbon, 2014, 80, 382-386.

111 C. Jin, H. Lan, L. Peng, K. Suenaga and S. Iijima, Phys. Rev. Lett., 2009, 102, 205501.

112 A. Lucotti, C. S. Casari, M. Tommasini, A. Li Bassi, D. Fazzi, V. Russo, M. Del Zoppo, C. Castiglioni, F. Cataldo, C. E. Bottani and G. Zerbi, Chem. Phys. Lett., 2009, 478, 45.

113 C. S. Casari, V. Russo, A. Li Bassi, C. E. Bottani, F. Cataldo, A. Lucotti, M. Tommasini, M. Del Zoppo, C. Castiglioni and G. Zerbi, Appl. Phys. Lett., 2007, 90, 013111.

114 S. Okada, M. Fujii and S. Hayashi, Carbon, 2011, 49, 4704. 115 F. Cataldo and C. S. Casari, J. Inorg. Organomet. Polym. Mater., 2007, 17, 641.

116 C. S. Casari, A. Li Bassi, A. Baserga, L. Ravagnan, P. Piseri, C. Lenardi, M. Tommasini, A. Milani, D. Fazzi, C. E. Bottani and P. Milani, Phys. Rev. B: Condens. Matter, 2008, 77, 195444.

117 M. M. Yildizhan, D. Fazzi, A. Milani, L. Brambilla, M. Del Zoppo, W. Chalifoux, R. R. Tykwinski and G. Zerbi, J. Chem. Phys., 2011, 134, 124512.

118 V. Cadierno and J. Gimeno, Chem. Rev., 2009, 109, 3512.

119 F. Cataldo, J. Inorg. Organomet. Polym. Mater., 2006, 16, 15.

120 M. Tommasini, A. Milani, D. Fazzi, A. Lucotti, C. Castiglioni, J. A. Januszewski, D. Wendinger and R. R. Tykwinski, J. Phys. Chem. C, 2014, 118, 2641526425.

121 D. Fazzi, F. Scotognella, A. Milani, D. Brida, C. Manzoni, E. Cinquanta, M. Devetta, L. Ravagnan, P. Milani, F. Cataldo, L. Lueer, R. Wannemacher, J. Cabanillas-Gonzalez, M. Negro, S. Stagira and C. Vozzi, Phys. Chem. Chem. Phys., 2013, 15, 9384.

122 A. D. Slepkov, F. A. Hegmann, Y. Zhao, R. R. Tykwinski and K. Kamada, J. Chem. Phys., 2002, 116, 3834-3840.

123 S. Eisler, A. D. Slepkov, E. Elliott, T. Luu, R. McDonald, F. A. Hegmann and R. R. Tykwinski, J. Am. Chem. Soc., 2005, 127, 2666-2676.

124 A. D. Slepkov, F. A. Hegmann, S. Eisler, E. Elliott and R. R. Tykwinski, J. Chem. Phys., 2004, 120, 6807-6810.

125 C. Castiglioni, M. Del Zoppo, P. Zuliani and G. Zerbi, Synth. Met., 1995, 74, 171-177.

126 M. Rumi, G. Zerbi, K. Müllen, G. Müller and M. Rehahn, J. Chem. Phys., 1997, 106, 24-34.

127 R. R. Tykwinski, W. Chalifoux, S. Eisler, A. Lucotti, M. Tommasini, D. Fazzi, M. Del Zoppo and G. Zerbi, Pure Appl. Chem., 2010, 82, 891-904. 
128 C. Castiglioni, M. Del Zoppo and G. Zerbi, Phys. Rev. B: Condens. Matter, 1996, 53, 13319-13325.

129 M. G. Kuzyk, Phys. Rev. Lett., 2000, 85, 1218-1221; erratum: M. G. Kuzyk, Phys. Rev. Lett., 2003, 90, 039902.

130 T. Luu, E. Elliott, A. D. Slepkov, S. Eisler, R. McDonald, F. A. Hegmann and R. R. Tykwinski, Org. Lett., 2005, 7, 51-54.

131 J. C. May, J. H. Lim, I. Biaggio, N. N. P. Moonen, T. Michinobu and F. Diederich, Opt. Lett., 2005, 30, 30573059.

132 E. Fazio, S. Patanè, L. D’Urso, G. Compagnini and F. Neri, Opt. Commun., 2012, 285, 2942.

133 A. Arendt, R. Kolkowski, M. Samoc and S. Szafert, Phys. Chem. Chem. Phys., 2015, 17, 13680-13688.

134 G.-L. Xu, C.-Y. Wang, Y.-H. Ni, T. G. Goodson III and T. Ren, Organometallics, 2005, 24, 3247-3254.

135 E. Fazio, L. D’Urso, G. Consiglio, A. Giuffrida, G. Compagnini, O. Puglisi, S. Patane, F. Neri and G. Forte, J. Phys. Chem. C, 2014, 118, 28812-28819.

136 M. Samoc, G. T. Dalton, J. A. Gladysz, Q. Zheng, Y. Velkov, H. Agren, P. Norman and M. G. Humphrey, Inorg. Chem., 2008, 47, 9946-9957.

137 M. Liu, V. I. Artyukhov, H. Lee, F. Xu and B. I. Yakobson, ACS Nano, 2013, 7(11), 10075-10082.

138 H. E. Troiani, M. Miki-Yoshida, G. A. Camacho-Bragado, M. A. L. Marques, A. Rubio, J. A. Ascencio and M. JoseYacaman, Nano Lett., 2003, 3, 751.

139 R. Landauer, IBM J. Res. Dev., 1957, 1, 223-231.

140 B. Larade, J. Taylor, H. Mehrez and H. Guo, Phys. Rev. B: Condens. Matter, 2001, 64, 075420.

141 Z. Crljen and G. Baranovic, Phys. Rev. Lett., 2007, 98, 116801.

142 W. Chen, et al., Phys. Rev. B: Condens. Matter, 2009, 80, 085410.

143 Z. Zanolli, G. Onida and J. C. Charlier, ACS Nano, 2010, 4, 5174.

144 C. Wang, A. S. Batsanov, M. R. Bryce, S. Martín, R. J. Nichols, S. J. Higgins, V. M. García-Suarez and C. J. Lambert, J. Am. Chem. Soc., 2009, 131, 15647-15654.

145 P. Moreno-García, M. Gulcur, D. Zsolt Manrique, T. Pope, W. Hong, V. Kaliginedi, C. Huang, A. S. Batsanov, M. R. Bryce, C. Lambert and T. Wandlowski, J. Am. Chem. Soc., 2013, 135, 12228-12240.

146 M. Gulcur, P. Moreno-García, X. Zhao, M. Baghernejad, A. S. Batsanov, W. Hong, M. R. Bryce and T. Wandlowski, Chem. - Eur. J., 2014, 20, 4653-4660.

147 S. Ballmann, W. Hieringer, D. Secker, Q. Zheng, J. A. Gladysz, A. Görling and H. B. Weber, Chem. Phys. Chem., 2010, 11, 2256-2260.
148 T. D. Yuzvinsky, et al., Nano Lett., 2006, 6, 2718.

149 B. Standley, W. Bao, H. Zhang, J. Bruck, C. Ning Lau and M. Bockrath, Nano Lett., 2008, 8(10), 3345-3349.

150 O. Cretu, et al., Nano Lett., 2013, 13(8), 3487-3493.

151 A. La Torre, A. Botello-Mendez, W. Baaziz, J.-C. Charlier and F. Banhart, Nat. Commun., 2015, 6, 6636.

152 C. Zhao, et al., J. Phys. Chem. C, 2011, 115, 13166.

153 E. Hobi, et al., Phys. Rev. B: Condens. Matter, 2010, 81, 201406.

154 Y. Wang, et al., Phys. Rev. B: Condens. Matter, 2007, 76, 165423.

155 C. Ataca, et al., Phys. Rev. B: Condens. Matter, 2011, 83, 235417.

156 R. Rivelino, et al., J. Phys. Chem. C, 2010, 114, 16367.

157 E. Cinquanta, et al., J. Chem. Phys., 2011, 135, 194501.

158 F. Cataldo, et al., J. Phys. Chem., 2010, 114B, 14834.

159 Y. Yamaguchi, et al., Chem. Phys. Lett., 2004, 388, 436.

160 A. L. Ivanovskii, Prog. Solid State Chem., 2013, 41, 1.

161 R. H. Baughman, H. Eckhardt and M. Kertesz, J. Chem. Phys., 1987, 87, 6687.

162 M. M. Haley, Angew. Chem., Int. Ed. Engl., 1997, 36, 835.

163 D. Malko, C. Neiss, F. Viñes and A. Görling, Phys. Rev. Lett., 2012, 108, 086804.

164 D. Malko, C. Neiss and A. Görling, Phys. Rev. B: Condens. Matter, 2012, 86, 045443.

165 V. N. Popov and P. Lambin, Phys. Rev. B: Condens. Matter, 2013, 88, 075427.

166 H. Bai, Y. Zhu, W. Qiao and Y. Huang, RSC Adv., 2011, 1, $768-775$.

167 V. R. Coluci, S. F. Braga, S. B. Legoas, D. S. Galvão and R. H. Baughman, Phys. Rev. B: Condens. Matter, 2003, 68, 035430.

168 V. R. Coluci, et al., Nanotechnology, 2004, 15, S142.

169 G. Li, Y. Li, H. Liu, Y. Guo, Y. Li and D. Zhu, Chem. Commun., 2010, 46, 3256-3258.

170 F. Diederich and M. Kivala, Adv. Mater., 2010, 22, 803812.

171 P. Rivera-Fuentes and F. Diederich, Angew. Chem., Int. Ed., 2012, 51, 2818-2828.

172 M. M. Haley, Pure Appl. Chem., 2008, 80, 519-532.

173 M. M. Haley, S. C. Brand and J. J. Pak, Angew. Chem., Int. Ed. Engl., 1997, 36, 835.

174 J. M. Kehoe, J. H. Kiley, J. J. English, C. A. Johnson, R. C. Petersen and M. M. Haley, Org. Lett., 2000, 2, 969.

175 M. Gholami, et al., Chem. Commun., 2009, 3038-3040.

176 Z. Li, M. Smeu, A. Rives, V. Maraval, R. Chauvin, M. A. Ratner and E. Borguet, Nat. Commun., 2015, 6, 6321.

177 P. B. Sorokin, H. Lee, L. Yu. Antipina, A. K. Singh and B. I. Yakobson, Nano Lett., 2011, 11(7), 2660-2665. 